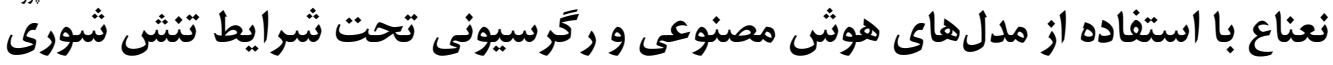

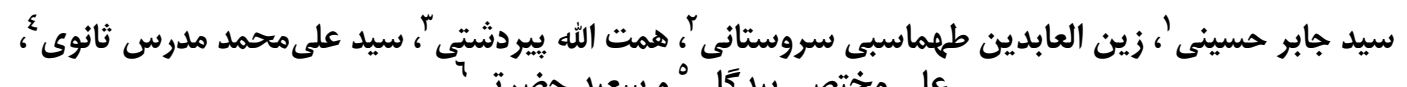

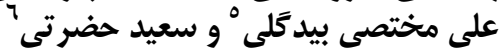

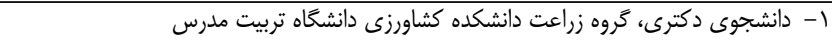

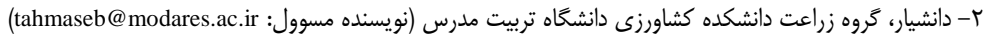

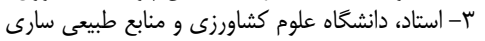

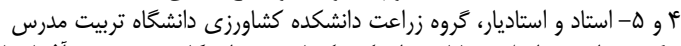

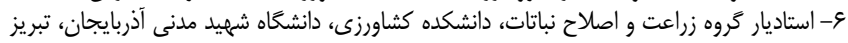
تاريخ يذيرش: تاريخ دريافت:

صفحه: Q9 ن تا VI

جكيده

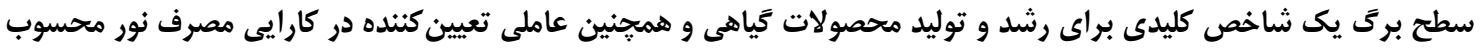

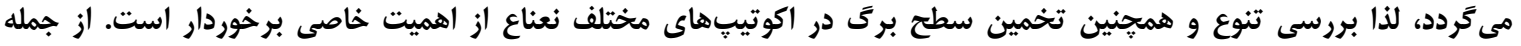

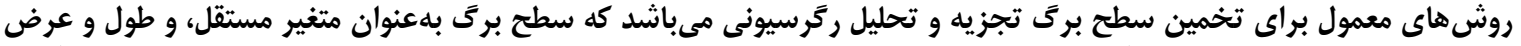

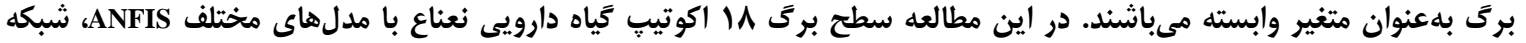

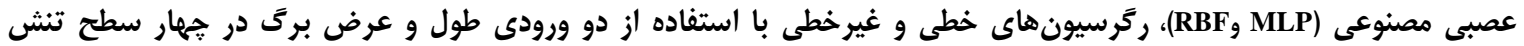
شورى (شاهد،

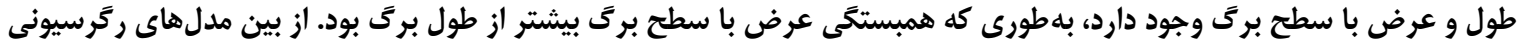

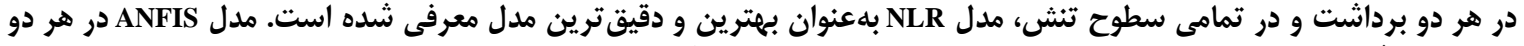

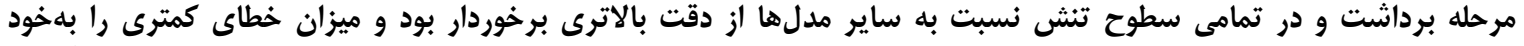

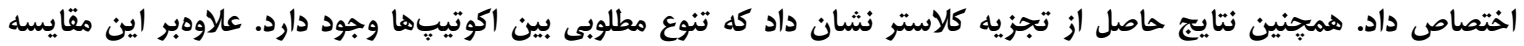

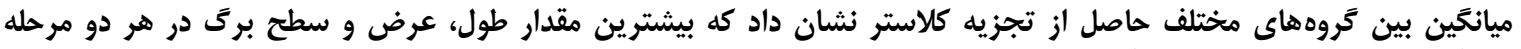

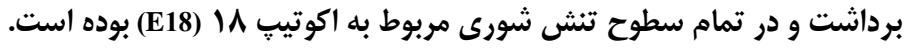

وازههاى كليدى: اكوتيڤ، تنش شورى، سطح برَى، نعناع، مدلهاى ركرسيونى

آبيارى است (V). بنابراين سطح برى گياهان بلشدت رشات رشد گياه و

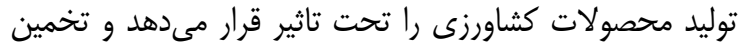

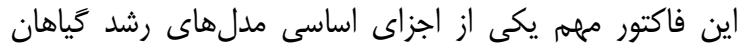

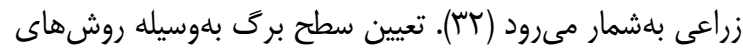

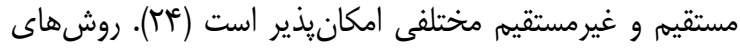

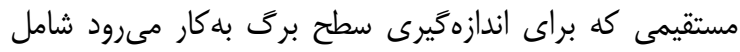

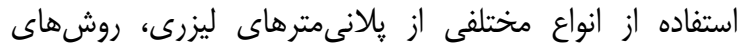

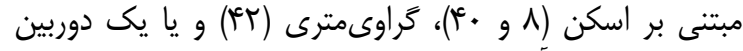

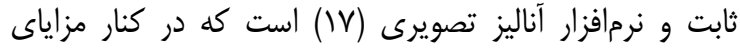

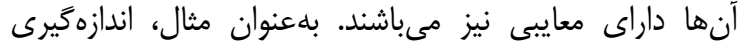

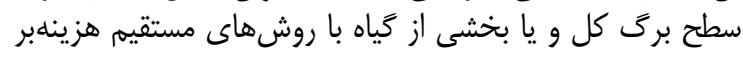

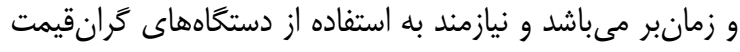

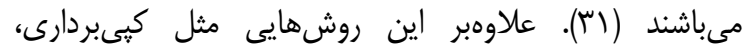

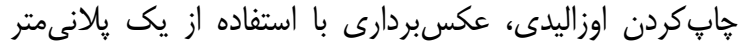

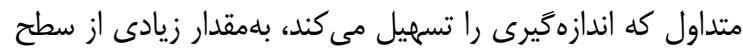

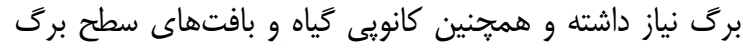

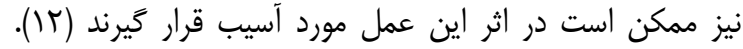

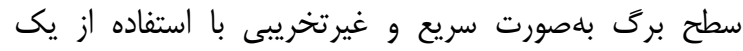

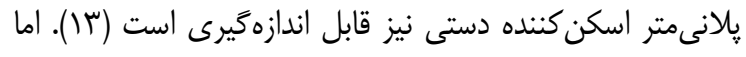

مقدمه

نعناع يكى از يُرمصرفترين گياهان دارويى است كه مقدار

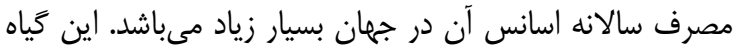

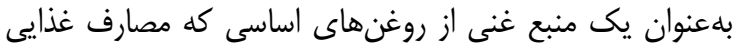

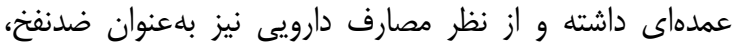

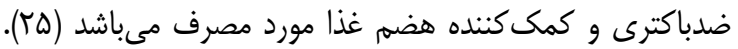

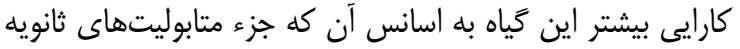

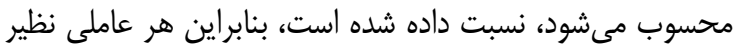

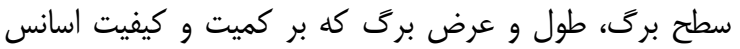

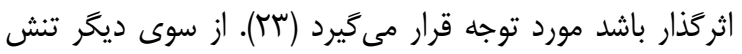

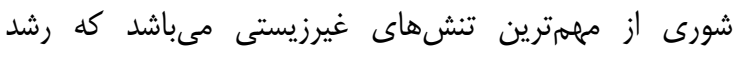

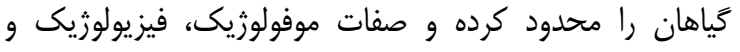

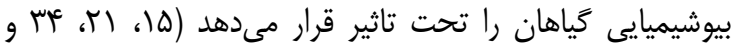

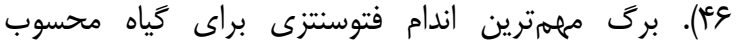

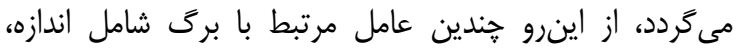

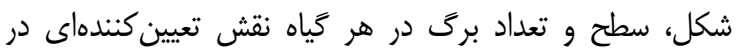

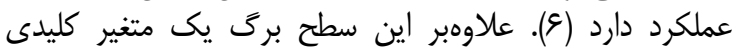

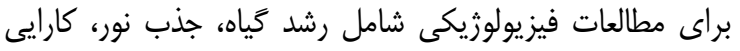

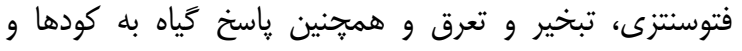




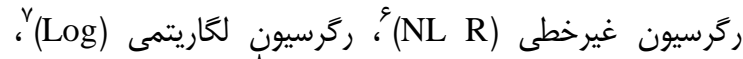

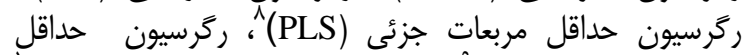

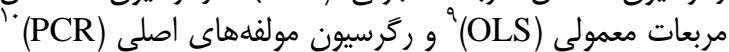
براى بيشيبنى ميزان يارامتر خروجى در مر مطالعات متعدد استفاده

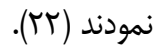

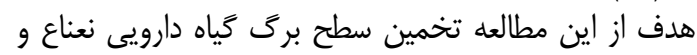

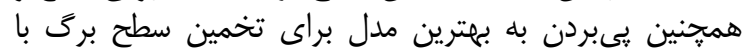

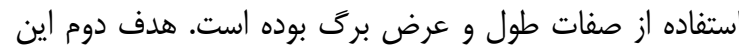

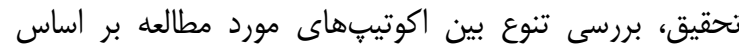
صفات مربوط به سطح برى با استفاده از تجزيه خوشهاى برى بوده

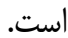

\section{مواد و روشها}

اين تحقيق در يثوهشكده زنتيكى و زيستفناوناوى كشاورزى

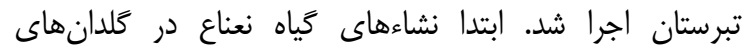

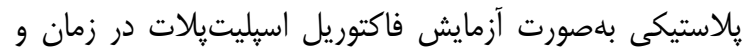

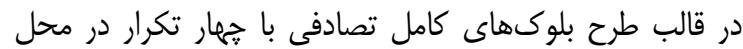

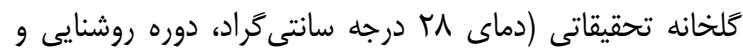

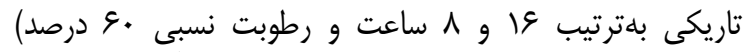

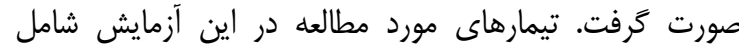

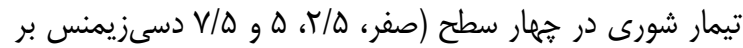

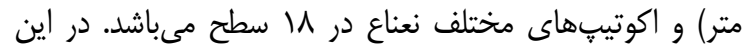

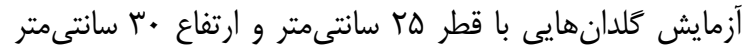

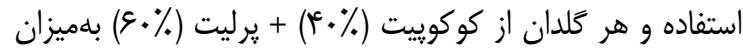

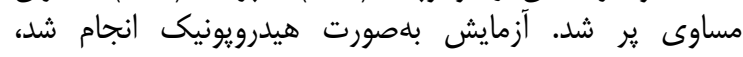

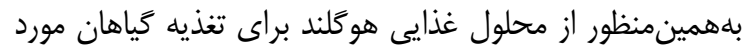

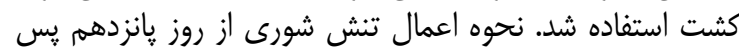

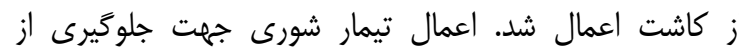

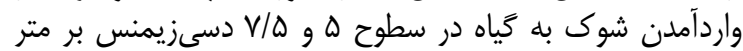

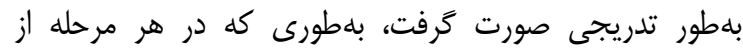

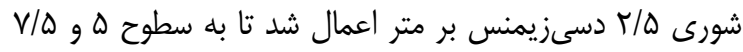

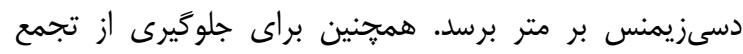

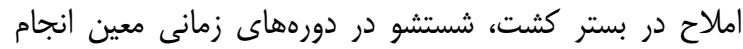

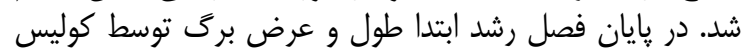

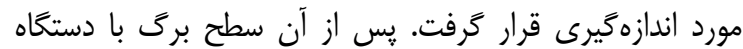

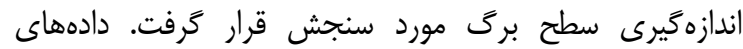

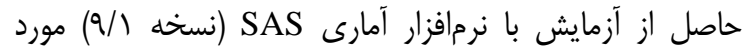

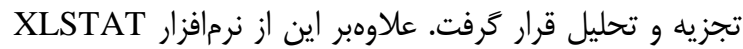

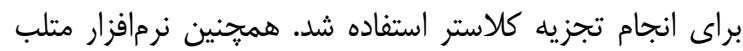

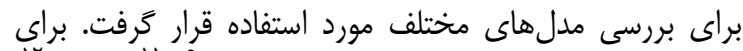

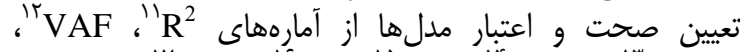
أم

كه اين آمارهها توسط روابط زير قابل محاسبه است (Tس.كT).

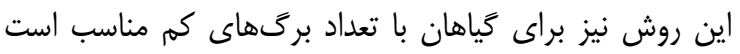

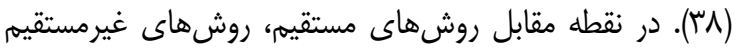

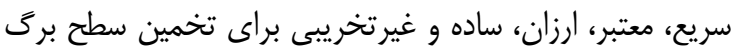

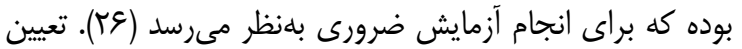

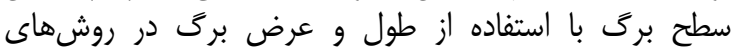

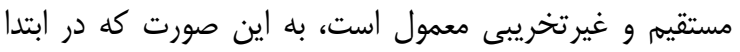

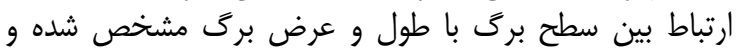

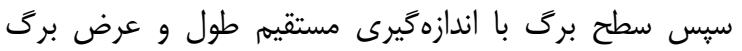

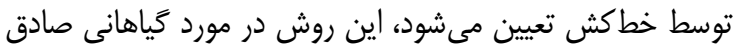

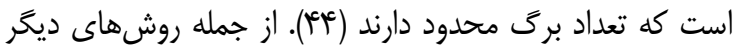

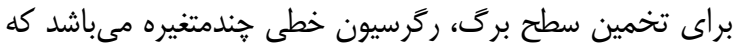

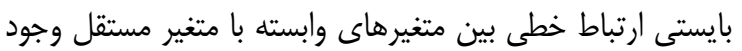

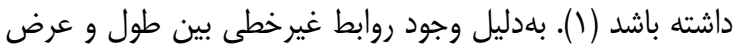

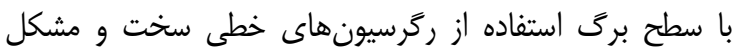

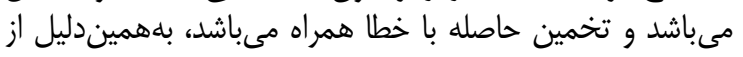

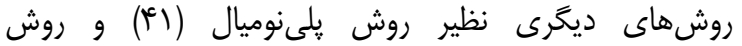

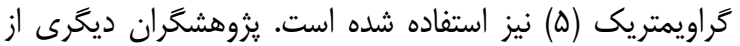

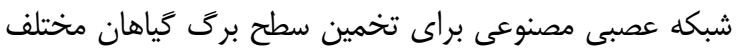

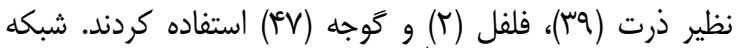

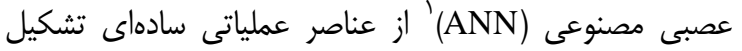

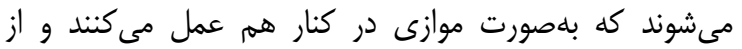

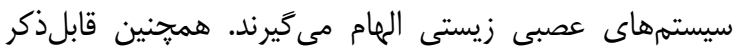

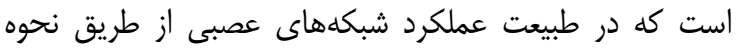

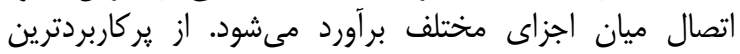

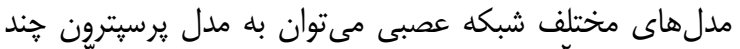

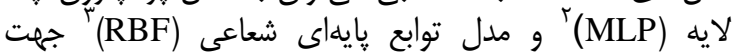

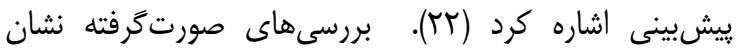

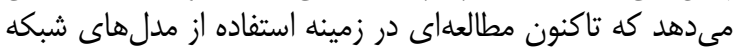

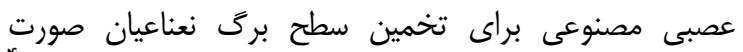

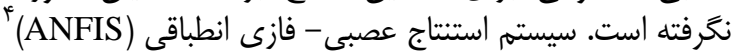

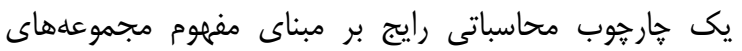

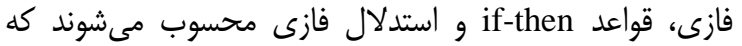

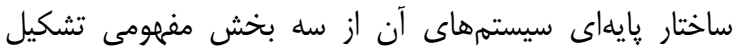

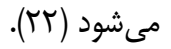

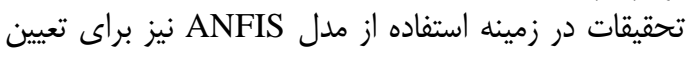

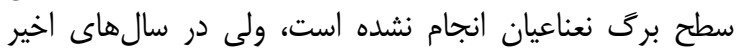

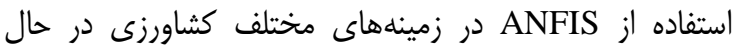

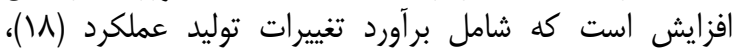

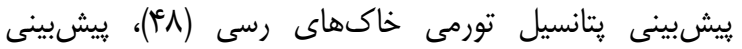

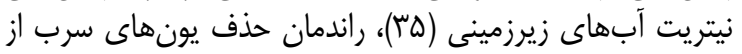

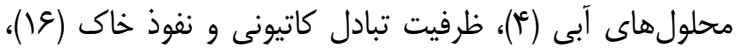

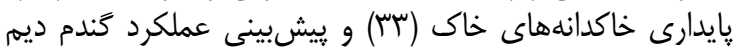

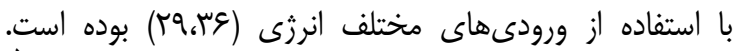

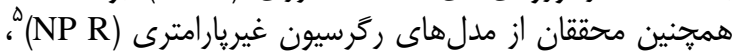

1- Artificial neural network

4- Adaptive neuro fuzzy inference system

7- Logarithmic regression

10- Principal component regression

13- Mean absolute percentage error

16- Bayesian information criteria

17- Akaike information criterion
3- Radial basis function

6- Nonlinear regression

9- Ordinary least squares regression

12- Variance account factor

15 - Relative percent difference
2- Multilayer perceptron

5- Nonparametric regression

11- Coefficient of determination

14- Root mean square error 


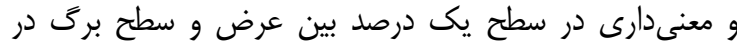

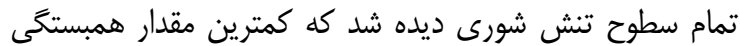

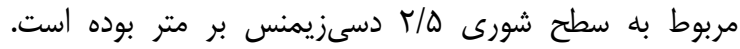

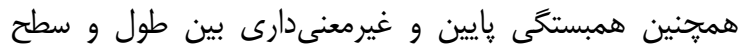

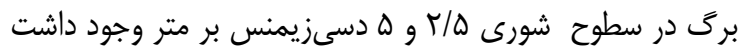

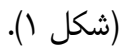
نتايج آناليز حساسيت نشان داد كه در هر دو برداشت، اهميت

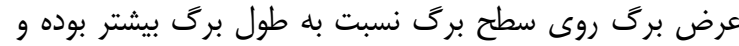

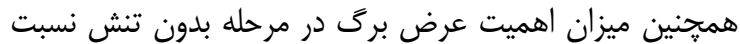

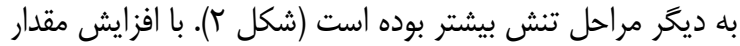

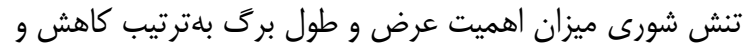

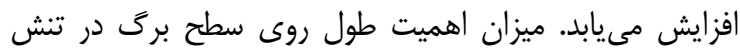

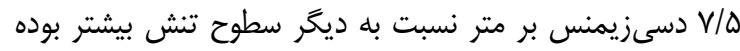

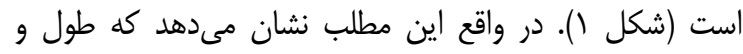

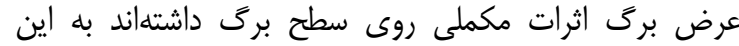
صورت كه با افزايش تنش بلهترتيب از اثر عرض برى برى برى بر سطح

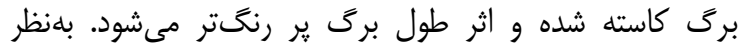

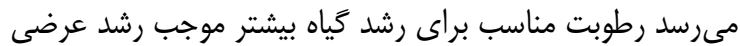

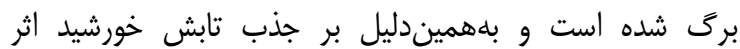

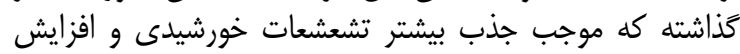

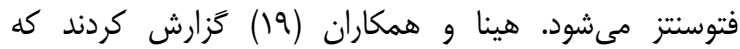

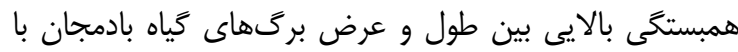
سطح برگ وجود داشته است.
(Equation 1) $\mathrm{VAF}=\left[1-\frac{\operatorname{Var}(P(s i)-M(s i)}{\operatorname{Var}(M(s i)}\right] \times 100$

(Equation 2) $\quad \mathrm{RMSE}=\sqrt[2]{\left\{\frac{\sum[P(s i)-M(s i)]^{2}}{n}\right\}}$

(Equation 3) $\mathrm{R} 2=1-\frac{\sum_{i=1}^{n}[M(s i)-P(s i)]^{2}}{\sum_{i=1}^{n}[M(s i)-A(s i)]^{2}}$

(Equation 4) $\mathrm{MAPE}=\frac{\sum_{i}^{N}\left|\frac{A i-P i}{A i}\right|}{N} \times 100$

(Equation 5) $\quad \mathrm{BIC}=(\mathrm{n}) \log (\mathrm{SSE} / \mathrm{n})+(\mathrm{K}) \log (\mathrm{n})$

(Equation 6) $\mathrm{AIC}=(\mathrm{n}) \log (\mathrm{SSE} / \mathrm{n})+2 \mathrm{p}$

(Equation 6) $\mathrm{RPD}=\frac{S D}{R M S E P}$

نتايج و بحث

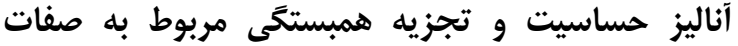

سطح با طول و عرض برى

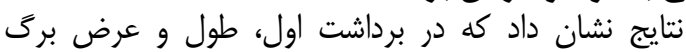

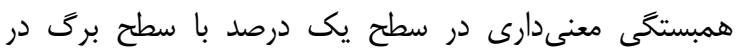

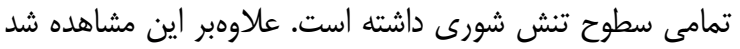

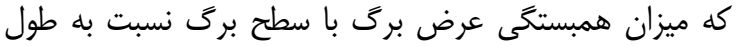

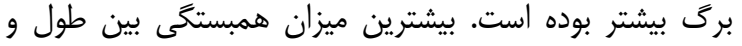

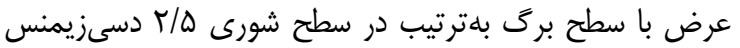

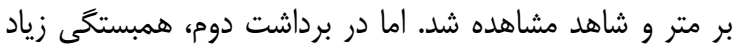

A

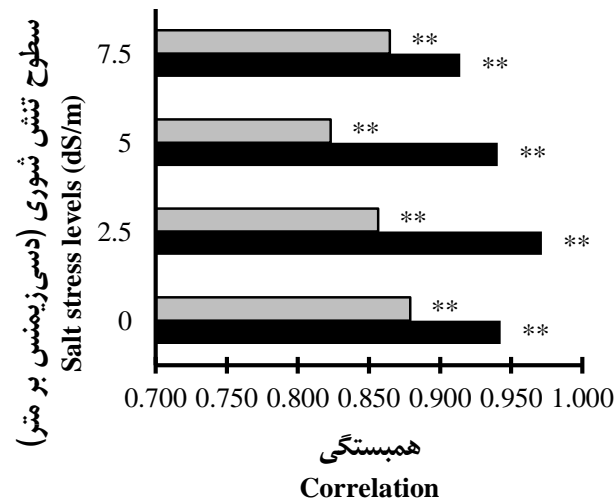

B

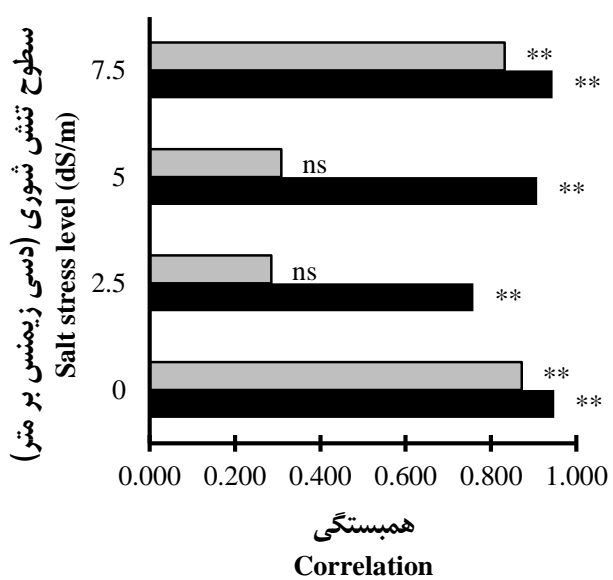

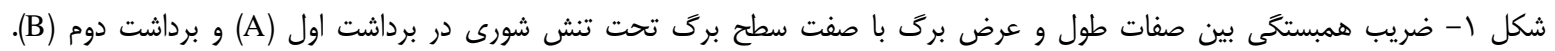

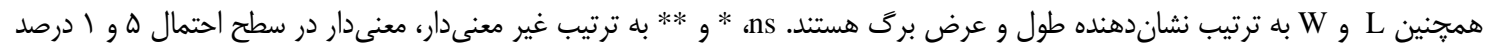

Figure 1. Correlation coefficient between length and width traits of leaf with leaf area under salt stress condition in first harvest (A) and second harvest (B). L and W related to length and width of leaf, respectively. Ns, * and **: are non-significant and significant at 5 and $1 \%$ probability levels, respectively. 

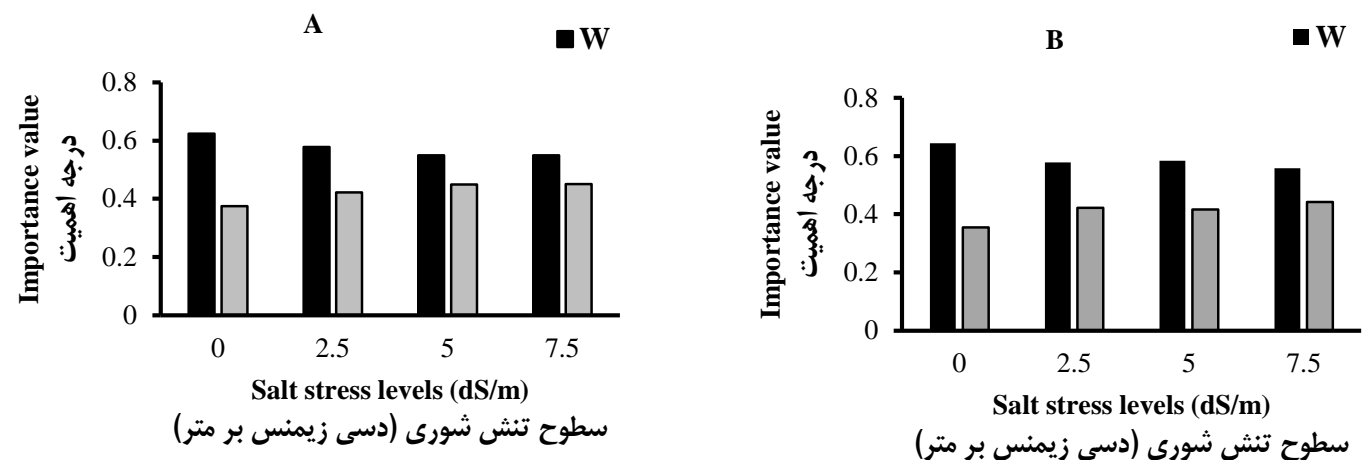

شكل r- نتايج آناليز حساسيت مربوط به صفات طول و عرض برگ تحت تنش شورى در برداشت اول (A) و برداشت دوم (B) Figure 2. The results of sensitivity analysis related to length and width traits under salt stress conditions in first harvest (A) and second harvest (B)

از سه شاخص طول، عرض و سطح برى و مبتنىبر تجزيه

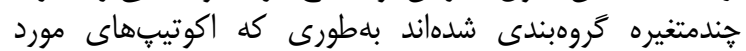

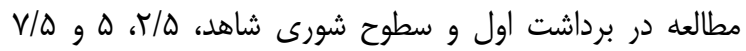

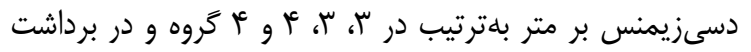

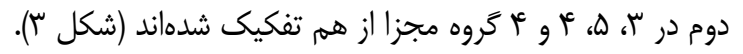

تجزيه كلاستر

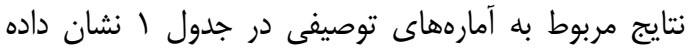

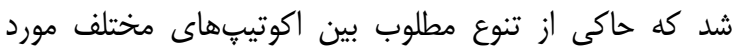

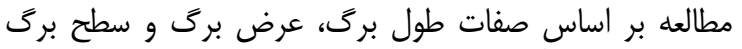

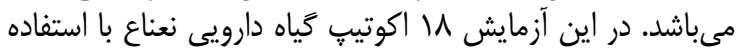

جدول (- آمارههاى توصيفى مربوط به صفات مختلف مورد مطالعه در 11 اكوتيب نعناع Table 1. Descriptive statistics related to different traits studied in 18 mint ecotypes

\begin{tabular}{|c|c|c|c|c|c|c|c|c|c|}
\hline \multirow{2}{*}{ تنش شورى (دسىزيمنس بر متر) } & \multirow{2}{*}{ 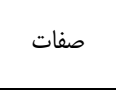 } & \multicolumn{4}{|c|}{ برداشت اول } & \multicolumn{4}{|c|}{ برداشت دوم } \\
\hline & & 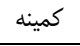 & بيشينه & ميانگين & انحراف معيار & كمينه & بيشينه & ميانگين & انحراف معيار \\
\hline \multirow[t]{3}{*}{ 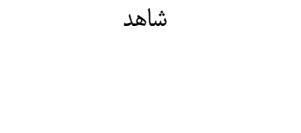 } & عرض برى & 1/ब91 & $F / \Delta \cdot \Delta$ & $\Gamma / \Delta \Delta V$ &. $\mid 991$ & $1 / 9 \Delta \Lambda$ & $r / \Delta V \Lambda$ & $r / 9 \mid \Lambda$ & . \\
\hline & طول برى & $1 / \cdot 10$ & $F / \Delta)$. & $r / g V{ }^{2}$ & - AYAD & $r / \cdot v \Delta$ & $r / \Delta V \Lambda$ & $r / V \mu$ & $\cdot /$ a f \\
\hline & سطح برى & $F / T \& \Lambda$ & $\mid F / N F \Lambda$ & $q / \Delta T$. & r/VQ. & $r / \Delta \cdot \Lambda$ & $15 / 991$ & १/^बT & r/VTr \\
\hline \multirow[t]{3}{*}{$r / \Delta$} & عرض برى & 1/ब9. & $r / q \cdot r$ & T/RMg &.$/ \% 9 \Delta$ & $1 / 94 \Delta$ & $\varphi / \Delta \cdot \Delta$ & T/NAN & $\cdot / V E V$ \\
\hline & طول برى & $r / V \Psi \Delta$ & $\Delta / \backslash \& \Delta$ & r/g/F & 年 & $\% / \cdot 10$ & $0 / .91$ & $F / I V T$ & $\cdot / V^{\mu} \cdot$ \\
\hline & سطح برى & 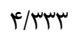 & $1 . / r r$. & V/DFF & 1/^rq & $r / r r$. & $\mid F / N A \Lambda$ & $N \cdot v G$ & $r / r r r$ \\
\hline \multirow[t]{3}{*}{$\Delta$} & عرض برى & 1/KTA & T/VVD & 1/9६9 & $\cdot /$ rqv & $1 / \uparrow \wedge \Delta$ & $r / \Delta \cdot \Delta$ & $r / \& \Delta$ & $\cdot|N|$ \\
\hline & طول برى & $r / T F A$ & $F / Q \Delta \wedge$ & $r / \cdots 1$ & $\cdot / F M$ & $r / \cdot 10$ & r/gr. & $r / 909$ & 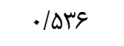 \\
\hline & سطح برى & $r / T V \Delta$ & १/द.. & $\Delta / \mathrm{V} v$ & I/AYa & $\Gamma / \Delta)$. & $\mid F / V F \Lambda$ & s/quq & $r / 9 \cdot r$ \\
\hline \multirow[t]{3}{*}{$V / \Delta$} & عرض برى & $1 / \cdot 1$. & T/KTD & 1/ब91 & $\cdot / \% \wedge 9$ & $1 / \cdot v \cdot$ & r/qYG & I/GAT & . FAT \\
\hline & طول برى & T & T/LT & $r / T \Delta 1$ & . IDST & $r / 4 \cdot r$ & F/fFe & $r / r \cdot q$ & $\cdot / \Delta \Delta V$ \\
\hline & سطح برى & $1 / 9 \vee \Delta$ & V/GYA & r/A)૬ & $1 / 419$ & $1 / \Lambda \Gamma \Delta$ & $9 / D / f$ & $r / 111$ & $1 / N \Gamma \Delta$ \\
\hline
\end{tabular}

E13) قرار گرفتند. از سويى ديگر نتايج حاصل از تجزيه كلاستر

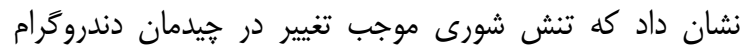

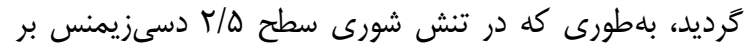

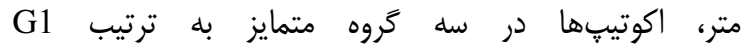

نتايج حاصل از تجزيه كلاستر نشان داد كه در برداشت

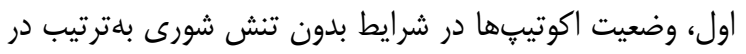
سه گروه E18, E10, E6, E7, E9, E5, E2, E3) G1) $\mathrm{E} 12, \mathrm{E} 4, \mathrm{E} 14, \mathrm{E} 16, \mathrm{E} 11, \mathrm{E} 17, \mathrm{E} 15, \mathrm{E} 8$, ) G3 و (E1) 
E13, ) G4 ،(E15, E8, E1, E5) G3 ، (E14, E16, E4, E11 (E10, E17, E6, E7, E9, E2, E3 صورت كرفته است (شكل نتايج مقايسه ميانكين بين كروههاى حاصل از تجزيه

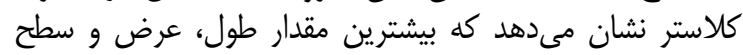

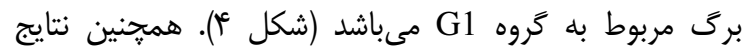

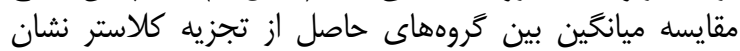

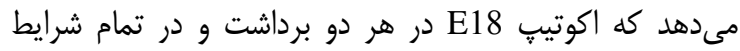

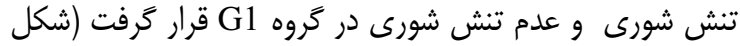

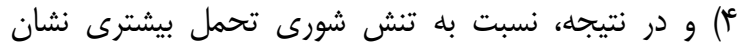

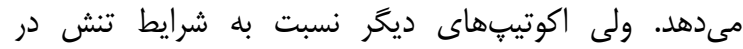

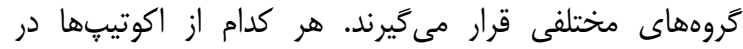

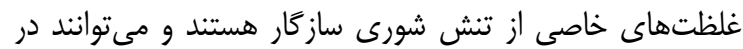

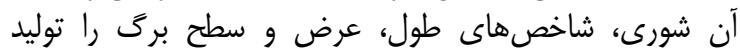

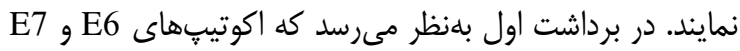

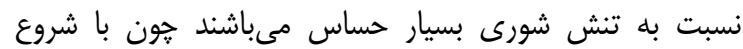

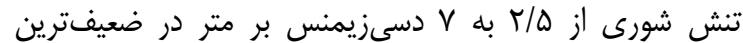

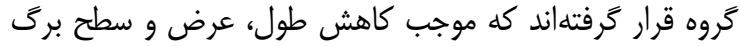

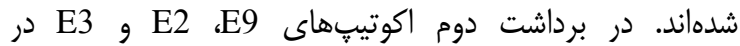
ضعيفترين گروه قرار داشتند كه نشان از حساسيت آنهائ آنها به

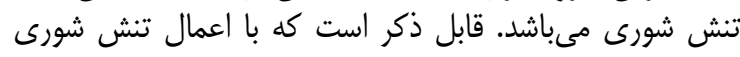

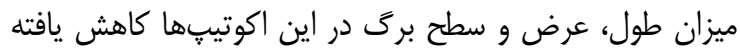

E15, E9, E3, ) G3 و (E16, E4) G2 ، (E18, E10, E13) (E17, E5, E12, E1, E8, E14, E2, E11, E6, E7 كَرفتند. همجنين نتايج در سطح شورى ه دسىزيمنس بر متر باترتيب E1)، E18) G14, E11, E12, E4, E16) G2)، E8, E15, E1, E5, E17, )G4 و (E13, E2, E9, E3, E10)

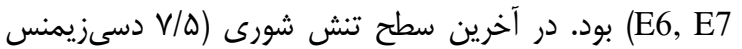

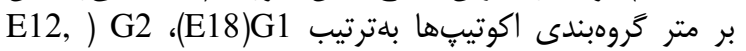
E3, ) G4 ،(E15, E8, E1, E5) G3 ،(E4, E11, E14, E16 (E7, E2, E6, E9, E13, E10, E17

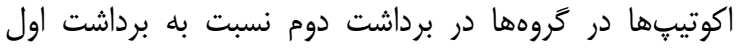

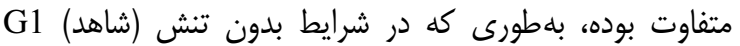
G3 (E1) G2 (E18, E6, E3, E10, E7, E9, E2 ,E5) (E12, E4, E14, E16, E17, E11, E8, E13, E15)

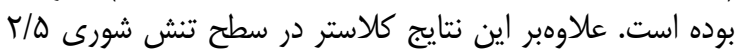

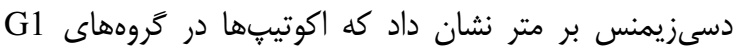
E12, E14, E16, E5, E6, E7, E1, ) G3 ،(E4) G2 ،(E18) (E2, E3, E9, E11, E17, E13, E15) G5 (E10) G4 (E8 قرار كَرفتند. از طرف ديكر در تنش شورى سطح ه دسىزيمنس

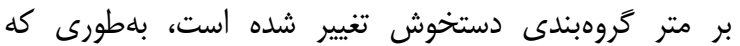

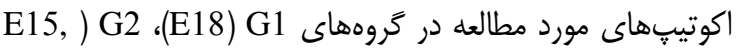
E14, E16, E9, ) G4، (E11, E4, E12) G3 ،(E13, E17

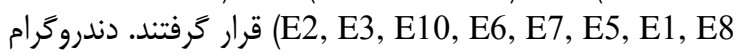

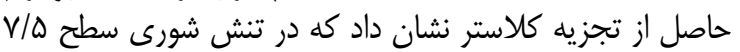

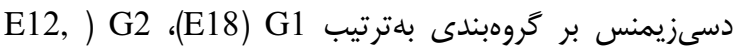




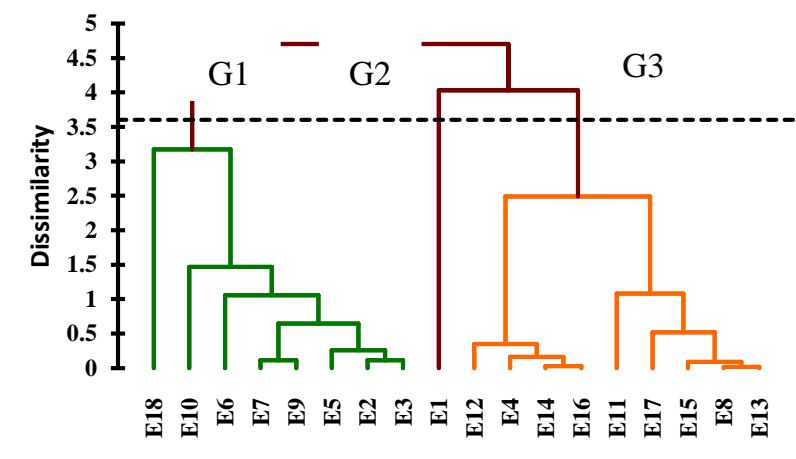

C

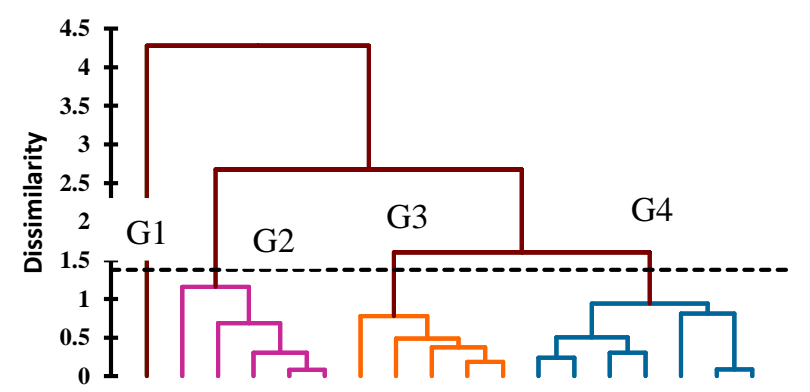

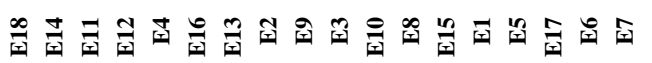

B

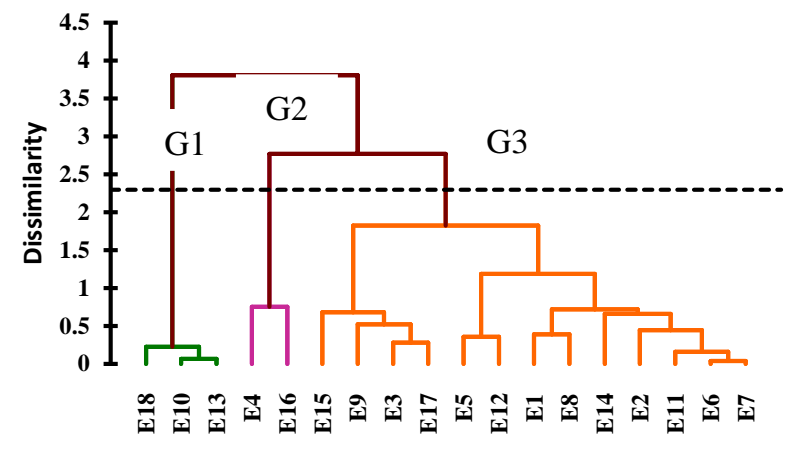

D

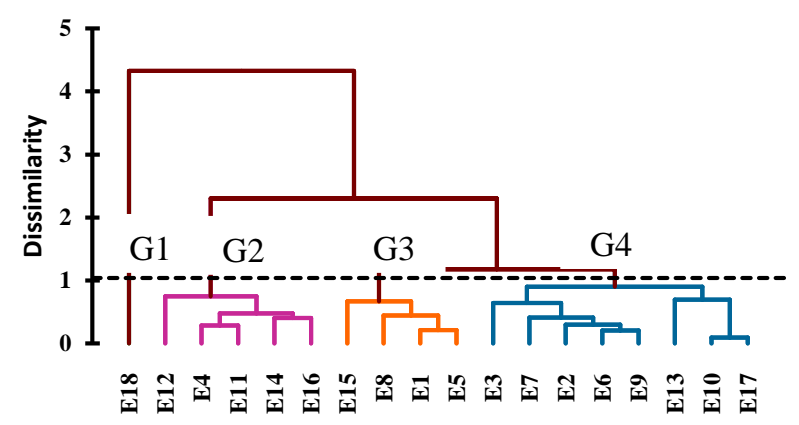

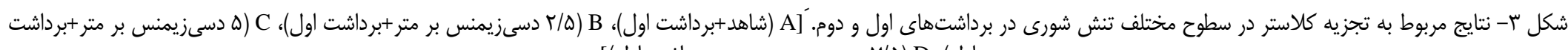

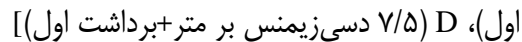

Figure 3. The results of cluster analysis under different salt stress levels in first and second harvest. [A (Control+first harvest), B (2.5 dS/m+first harvest), C (5 dS/m+first harvest), D (7.5 dS/m+first harvest)] 
E

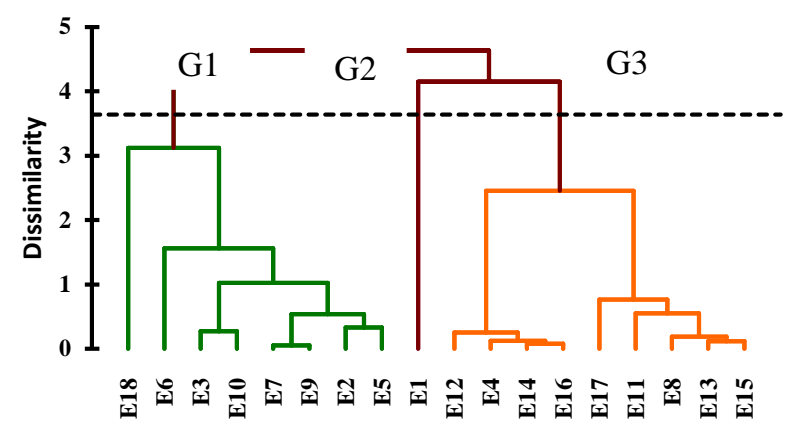

G

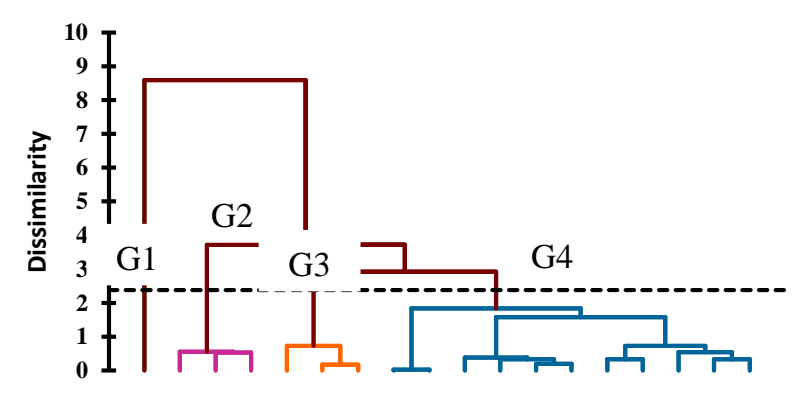

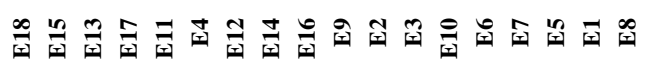

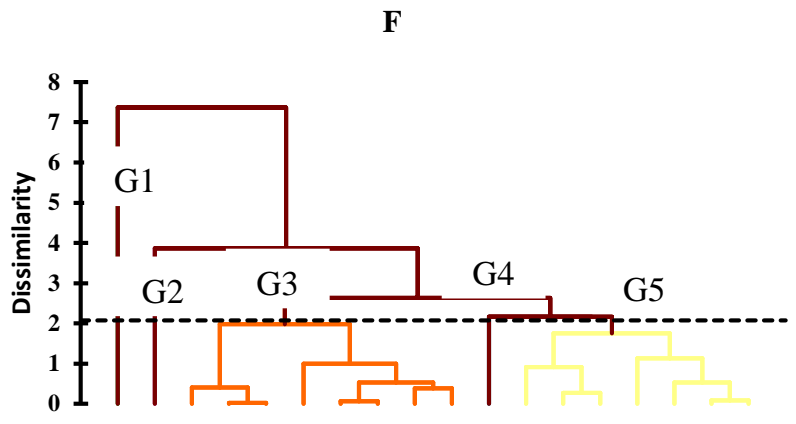

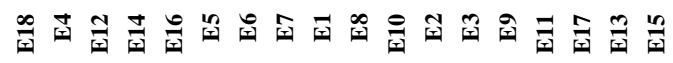

$\mathbf{H}$

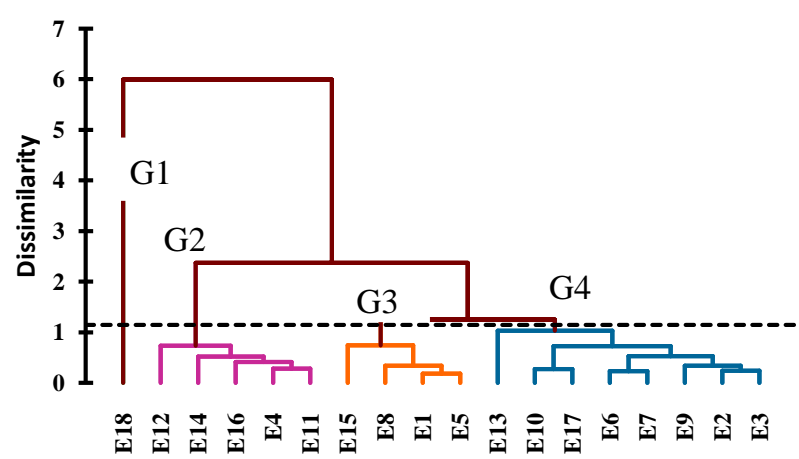

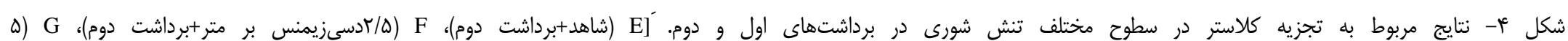

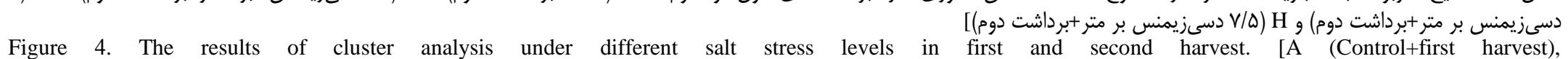

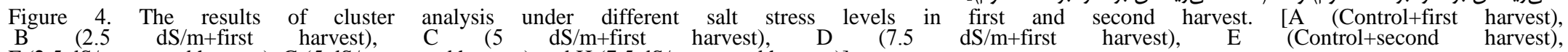
$\mathrm{F}$ (2.5 dS/m+second harvest), G (5 dS/m+second harvest) and $\mathrm{H}$ (7.5 dS/m+second harvest)] 


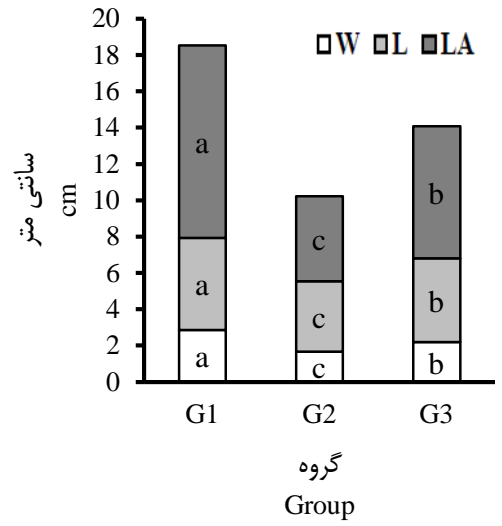

$\mathbf{E}$

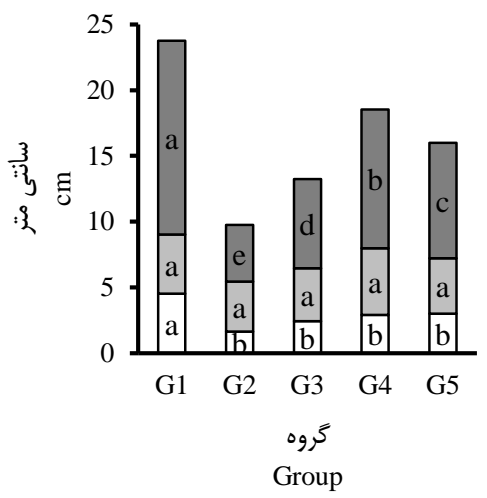

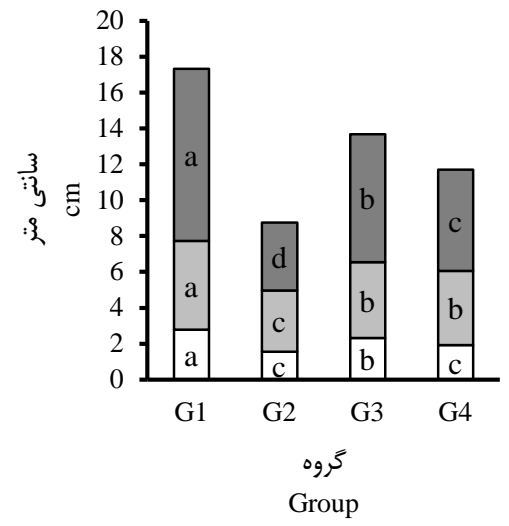

H

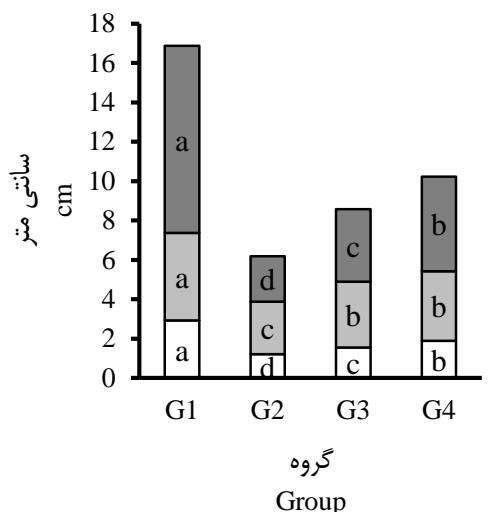

$\mathbf{C}$

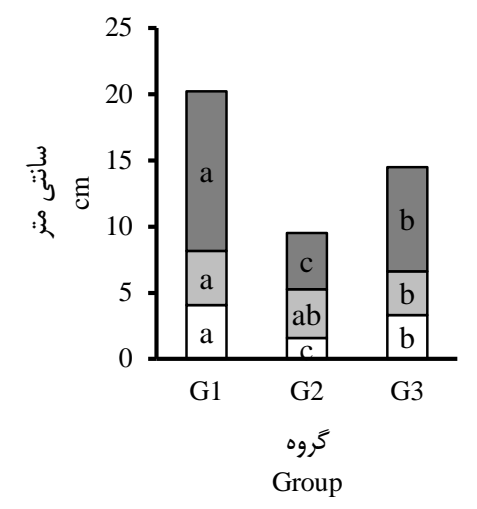

$\mathbf{F}$

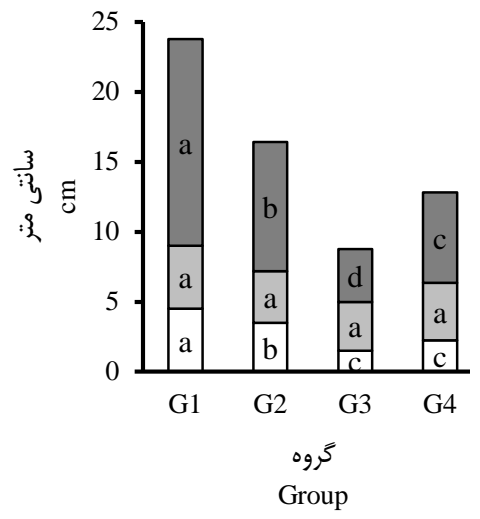

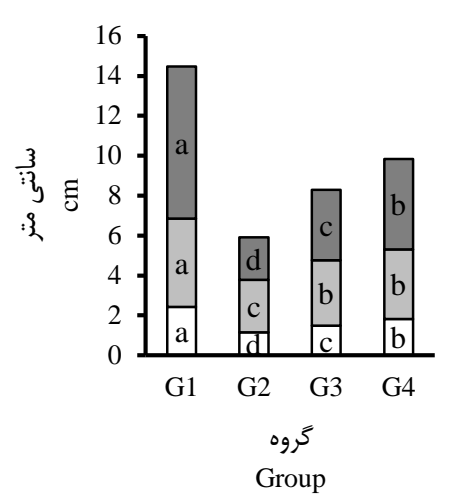

G

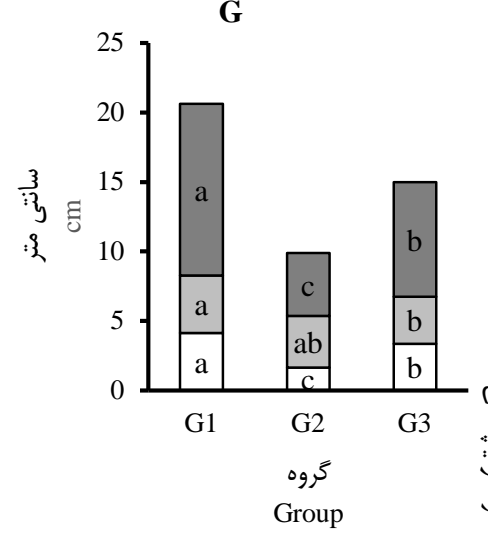

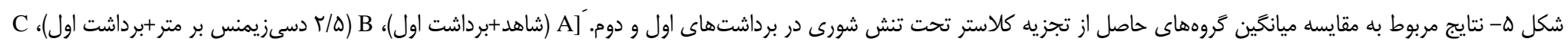

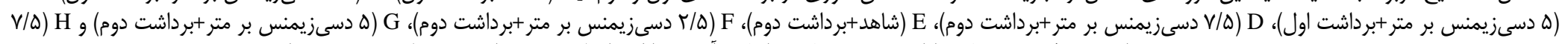

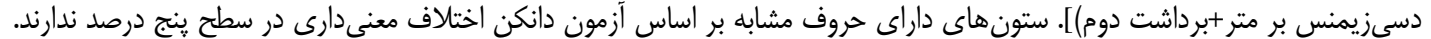

Figure 5. The results of mean comparison related to different groups of cluster analysis under salt stress condition in first and second harvest. [A (Control+first harvest), B (2.5 dS/m+first harvest), C (5 dS/m+first harvest), D ( $7.5 \mathrm{dS} / \mathrm{m}+$ first harvest), E (Control+second harvest), F ( $2.5 \mathrm{dS} / \mathrm{m}+\mathrm{second}$ harvest), G (5 dS/m+second harvest) and $\mathrm{H}$ (7.5 dS/m+second harvest)]. At $\alpha=5 \%$ based on Duncan test, means with similar letters in each column are not significantly different. 
مدل معرفى شد (R2=0.9849). بر مان طبق مطالعه هينا و همكاران

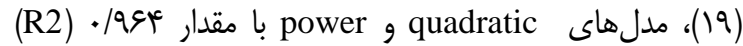

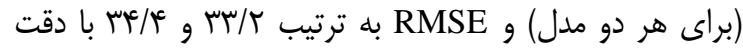

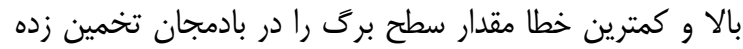

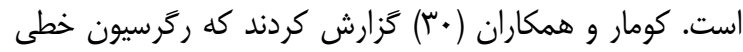

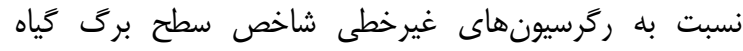
خاركيل (Durio zibethinus) را دقيقتر تخائ تخمين زده است. در

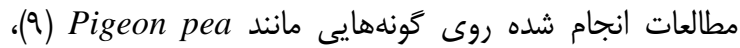

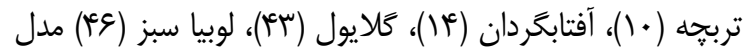

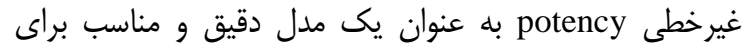

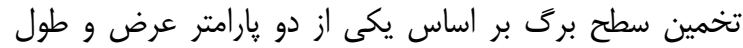

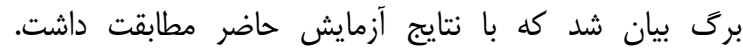

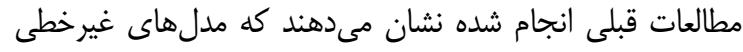

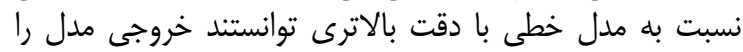

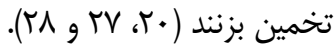

تخمين سطح بركى با استفاده از مدلهاى ركتر سيونى

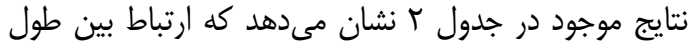

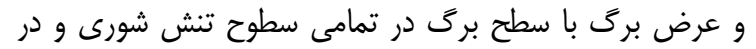

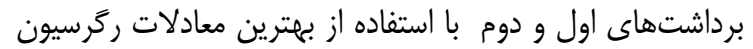

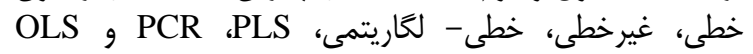

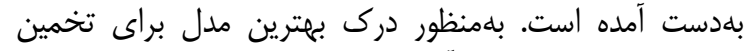

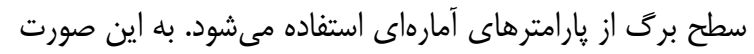

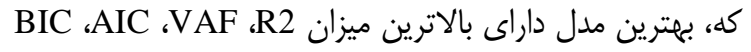

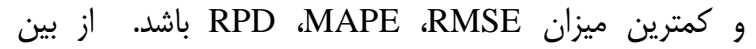
مدلهاى ركرسيونى در هر دو برداشت و در تمامى سطوح تنش،

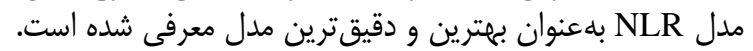

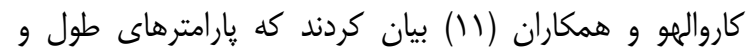
Crotalaria juncea عرض برى كارو براى تخمين سطح بران

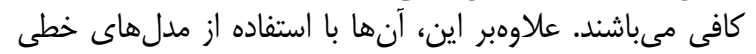

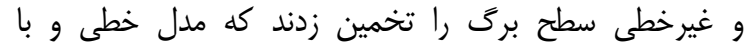

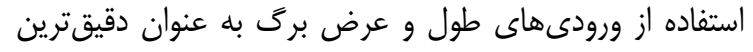

جدول r- نتايج مربوط به تخمين سطح برى اكوتيٍهاى مختلف نعناع با استفاده از مدلهاى مختلف ركر سيونى Table 2. Results related to estimation of leaf area for different mint ecotypes using different regression models

\begin{tabular}{|c|c|c|c|}
\hline 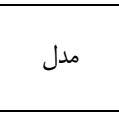 & $\begin{array}{l}\text { شورى } \\
\text { (dS/m) }\end{array}$ & برداشت دوم & برداشت اول \\
\hline \multirow[t]{4}{*}{ PLS } & . & $\mathrm{LA}=-10.179+2.819 * \mathrm{~W}+2.658 * \mathrm{~L}$ & $\mathrm{LA}=-9.838+2.7745 * \mathrm{~W}+2.611 * \mathrm{~L}$ \\
\hline & $r / \Delta$ & $\mathrm{LA}=-7.293+3.366 * \mathrm{~W}+1.586 * \mathrm{~L}$ & $\mathrm{LA}=-6.997+3.320 * \mathrm{~W}+1.543 * \mathrm{~L}$ \\
\hline & $\Delta$ & $\mathrm{LA}=-5.615+2.931 * \mathrm{~W}+1.405 * \mathrm{~L}$ & $\mathrm{LA}=-5.349+2.883 * \mathrm{~W}+1.362 * \mathrm{~L}$ \\
\hline & $V / \Delta$ & $\mathrm{LA}=-3.924+2.378 * \mathrm{~W}+1.221 * \mathrm{~L}$ & $\mathrm{LA}=-3.702+2.331 * \mathrm{~W}+1.176 * \mathrm{~L}$ \\
\hline \multirow[t]{4}{*}{ PCR } & . & $\mathrm{LA}=-10.179+2.819 * \mathrm{~W}+2.658 * \mathrm{~L}$ & $\mathrm{LA}=-9.838+2.7745 * \mathrm{~W}+2.611 * \mathrm{~L}$ \\
\hline & $r / \Delta$ & $\mathrm{LA}=-7.293+3.366 * \mathrm{~W}+1.586 * \mathrm{~L}$ & $\mathrm{LA}=-6.997+3.320 * \mathrm{~W}+1.543 * \mathrm{~L}$ \\
\hline & $\Delta$ & $\mathrm{LA}=-5.615+2.931 * \mathrm{~W}+1.405 * \mathrm{~L}$ & $\mathrm{LA}=-5.349+2.883 * \mathrm{~W}+1.362 * \mathrm{~L}$ \\
\hline & $V / \Delta$ & $\mathrm{LA}=-3.924+2.378 * \mathrm{~W}+1.221 * \mathrm{~L}$ & $\mathrm{LA}=-3.702+2.331 * \mathrm{~W}+1.176 * \mathrm{~L}$ \\
\hline \multirow[t]{4}{*}{ OLS } & • & $\mathrm{LA}=-10.179+2.819 * \mathrm{~W}+2.658 * \mathrm{~L}$ & $\mathrm{LA}=-9.838+2.7745 * \mathrm{~W}+2.611 * \mathrm{~L}$ \\
\hline & $r / \Delta$ & $\mathrm{LA}=-7.293+3.366 * \mathrm{~W}+1.586 * \mathrm{~L}$ & $\mathrm{LA}=-6.997+3.320 * \mathrm{~W}+1.543 * \mathrm{~L}$ \\
\hline & $\Delta$ & $\mathrm{LA}=-5.615+2.931 * \mathrm{~W}+1.405 * \mathrm{~L}$ & $\mathrm{LA}=-5.349+2.883 * \mathrm{~W}+1.362 * \mathrm{~L}$ \\
\hline & $V / \Delta$ & $\mathrm{LA}=-3.924+2.378 * \mathrm{~W}+1.221 * \mathrm{~L}$ & $\mathrm{LA}=-3.702+2.331 * \mathrm{~W}+1.176 * \mathrm{~L}$ \\
\hline \multirow[t]{4}{*}{$\begin{array}{c}\text { Linear } \\
\text { regression }\end{array}$} & $\cdot$ & $\mathrm{LA}=-10.179+2.819 * \mathrm{~W}+2.658 * \mathrm{~L}$ & $\mathrm{LA}=-9.838+2.7745 * \mathrm{~W}+2.611 * \mathrm{~L}$ \\
\hline & $r / \Delta$ & $\mathrm{LA}=-7.293+3.366 * \mathrm{~W}+1.586 * \mathrm{~L}$ & $\mathrm{LA}=-6.997+3.320 * \mathrm{~W}+1.543 * \mathrm{~L}$ \\
\hline & $\Delta$ & $\mathrm{LA}=-5.615+2.931 * \mathrm{~W}+1.405 * \mathrm{~L}$ & $\mathrm{LA}=-5.349+2.883 * \mathrm{~W}+1.362 * \mathrm{~L}$ \\
\hline & $V / \Delta$ & $\mathrm{LA}=-3.924+2.378 * \mathrm{~W}+1.221 * \mathrm{~L}$ & $\mathrm{LA}=-3.702+2.331 * \mathrm{~W}+1.176 * \mathrm{~L}$ \\
\hline \multirow{4}{*}{$\begin{array}{l}\text { Log-linear } \\
\text { regression }\end{array}$} & $\cdot$ & $\mathrm{LA}=\exp (0.259+0.319 * \mathrm{~W}+0.223 * \mathrm{~L})$ & $\mathrm{LA}=\exp (0.225+0.325 * \mathrm{~W}+0.2263 * \mathrm{~L})$ \\
\hline & $r / \Delta$ & $\mathrm{LA}=\exp (0.069+0.418 * \mathrm{~W}+0.213 * \mathrm{~L})$ & $\mathrm{LA}=\exp (0.002+0.429 * \mathrm{~W}+0.216 * \mathrm{~L})$ \\
\hline & $\Delta$ & $\mathrm{LA}=\exp (-0.146+0.460 * \mathrm{~W}+0.238 * \mathrm{~L})$ & $\mathrm{LA}=\exp (-0.195+0.475 * \mathrm{~W}+0.242 * \mathrm{~L})$ \\
\hline & $V / \Delta$ & $\mathrm{LA}=\exp (-0.518+0.538 * \mathrm{~W}+0.291 * \mathrm{~L})$ & $\mathrm{LA}=\exp (-0.576+0.558 * \mathrm{~W}+0.296 * \mathrm{~L})$ \\
\hline \multirow[t]{4}{*}{$\begin{array}{l}\text { Nonlinear } \\
\text { regression }\end{array}$} & . & $\mathrm{LA}=-2.950+2.241 * \mathrm{~W}-0.692 * \mathrm{~L}+0.086 * \mathrm{~W}^{\wedge} 2+0.435 * \mathrm{~L}^{\wedge} 2$ & $\mathrm{LA}=-2.783+2.203 * \mathrm{~W}-0.718 * \mathrm{~L}+0.086 * \mathrm{~W}^{\wedge} 2+0.440 * \mathrm{~L}^{\wedge} 2$ \\
\hline & $r / \Delta$ & $\mathrm{LA}=-2.401+1.195 * \mathrm{~W}+0.515 * \mathrm{~L}+0.463 * \mathrm{~W}^{\wedge} 2+0.116 * \mathrm{~L}^{\wedge} 2$ & $\mathrm{LA}=-2.196+1.149 * \mathrm{~W}+0.467 * \mathrm{~L}+0.475 * \mathrm{~W}^{\wedge} 2+0.118 * \mathrm{~L}^{\wedge} 2$ \\
\hline & Q & $\mathrm{LA}=-2.273+1.011 * \mathrm{~W}+0.704 * \mathrm{~L}+0.451 * \mathrm{~W}^{\wedge} 2+0.086 * \mathrm{~L}^{\wedge} 2$ & $\mathrm{LA}=-2.159+1.028 * \mathrm{~W}+0.661 * \mathrm{~L}+0.449 * \mathrm{~W}^{\wedge} 2+0.088 * \mathrm{~L}^{\wedge} 2$ \\
\hline & $V / \Delta$ & $\mathrm{LA}=-0.508+1.277 * \mathrm{~W}-0.317 * \mathrm{~L}+0.300 * \mathrm{~W}^{\wedge} 2+0.231 * \mathrm{~L}^{\wedge} 2$ & $\mathrm{LA}=-0.470+1.269 * \mathrm{~W}-0.320 * \mathrm{~L}+0.300 * \mathrm{~W}^{\wedge} 2+0.229 * \mathrm{~L}^{\wedge} 2$ \\
\hline
\end{tabular}




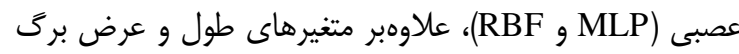

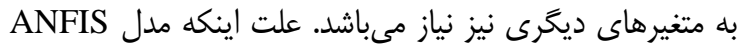

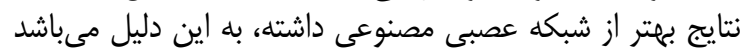

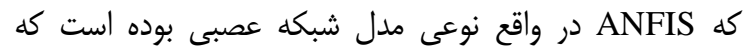

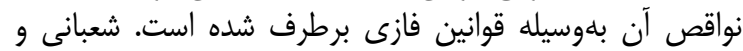

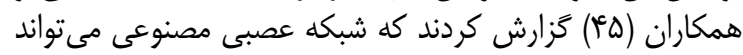

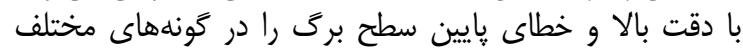

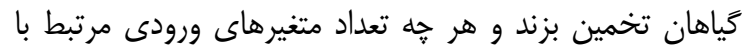

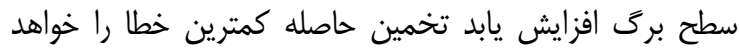

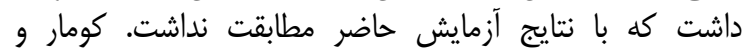

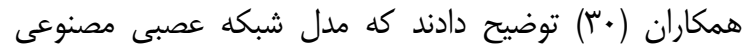

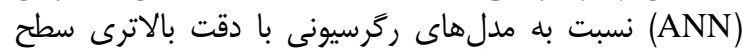

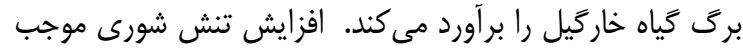

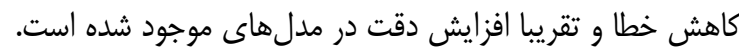

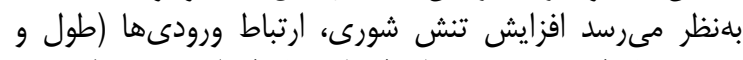

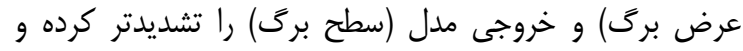

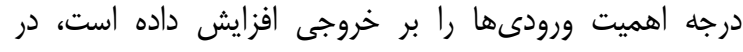

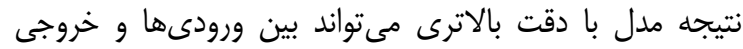

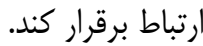

مقايسه مدلهاى رترسيونى و غير تركيونى براى تخمين سطح برى مدلى شاخص هاى عملكرد مدلها در برداشت اشتهاى اول و دوم در

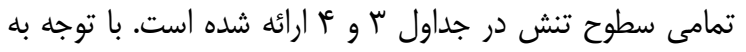

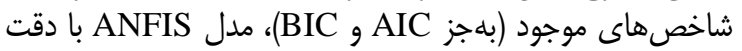

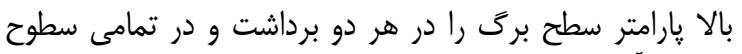

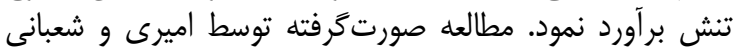

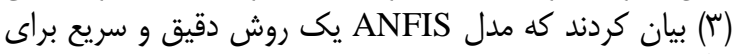

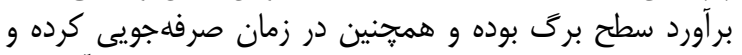

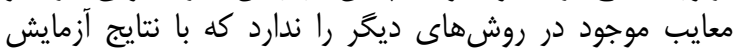

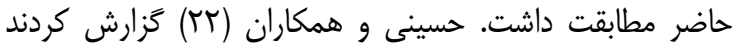
كه ANFIS با دقت بالايى توانست خروجى مدان مدل (فسفر خاك)

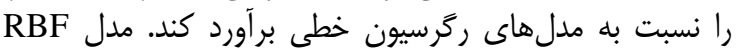

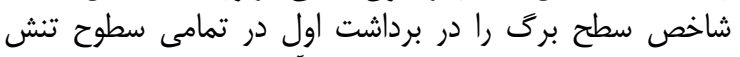

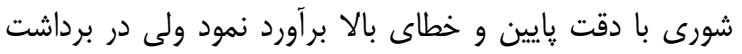

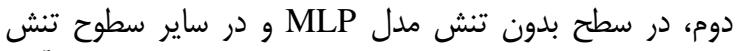

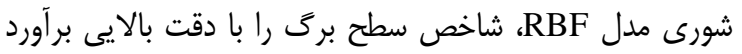

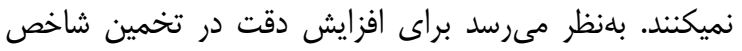
سطح برك در گياه دارويى نعناع با استفاده از مدلهاى درئ شبكه

جدول س- شاخصهاى عملكرد (RPD, AIC, BIC, RMSE, VAF, MAPE and R2) مربوط به مدل در برداشت اول Table 3. Performance indices (RPD, AIC, BIC, RMSE, VAF, MAPE and R2) for models in first harvest

\begin{tabular}{|c|c|c|c|c|c|c|c|c|c|c|c|}
\hline $\begin{array}{l}\text { شورى } \\
\text { (dS/m) }\end{array}$ & شملكرد & PLS & PCR & OLS & LR & $\log R$ & NL R & NP R & MLP & RBF & ANFIS \\
\hline \multirow[t]{7}{*}{0} & R2 &.$/ 919$ &.$/ 9 \wedge \varepsilon$ &.$/ Q \wedge \varepsilon$ &.$/ 9 \wedge \varepsilon$ &.$/ 9 \vee \Delta$ &.$/ 911$ &.$/ 99$. &.$/ 911$ &.$/ 9 \mathrm{VI}$ &.$/ 991$ \\
\hline & VAF & $99 / \Delta F F$ & १९/DFF & १৭/DT। & ११/DFF & $q V / \cdot r$. & १৭/४. ए & ৭৭/А५ळ & १৭/द५ & qর/द八 & १৭/Аৎ૬ \\
\hline & MAPE & $r / r \cdot 1$ & $r / r \cdot 1$ & T/TFe & $r / r \cdot 1$ & $4 / .11$ & r/৭DV & $r / r \cdot r$ & T/VFV & Ifle & $r / \Delta 19$ \\
\hline & RMSE & . MFe & . MFe & سمL/ & . TKF & . $/ \Lambda \mu$. & . TKF & . /TTD & $\cdot / 4 \cdot q$ & . IATD & $\cdot / r \cdot r$ \\
\hline & RPD & $N / N<q$ & N/NFq & N/gIr & $N / N \& q$ & r/Al. & $|r / 4| r$ & س & $9 / 9.8$ & F/NEV & $|Q / T V|$ \\
\hline & AIC & $19 N / r \& D$ & $19 N / T \& D$ & $19 N / 194$ & $19 N / T \& D$ & $r \cdot r / \Lambda \vee G$ & 199/ArG & r../Fr. & $199 / K_{N}$ & $198 / v 9 \%$ & $r \cdot 1 / \pi \Delta r$ \\
\hline & BIC & $r \cdot N / N r$ & $r \cdot N \cdot N r$ & $r \cdot N \cdot N=11$ & $r \cdot N / N r$ & سو/سוr & r.q/gkr & $r \mid \cdot / r+\Lambda$ & $r \cdot q / 1 . r$ & $r \cdot s / s \mid \omega$ & $r \mid l / l s 9$ \\
\hline \multirow[t]{7}{*}{$r / \Delta$} & R2 & $\cdot / 9 \wedge r$ & $\cdot / 911$ &.$/ Q \Lambda Y$ & $\cdot / Q \Lambda T$ & $\cdot / 91$. & $\cdot / 9 \wedge \Delta$ & $\cdot / 9 M M$ & $\cdot / q \wedge f$ & $\cdot / 999$ &.$/ 919$ \\
\hline & VAF & ११/द्र & ११/Fै & $99 / 9 \cdot 0$ & $99 / 919$ & $99 / V^{+1}$ & १৭/А & ११/१५. & $99 / v 91$ & ৭৭/•^ & १৭/१४১ \\
\hline & MAPE & T/VYG & $r / \Lambda \cdot \Delta$ & T/VF. & T/VAV & T/TVT & t/VQT & T/FAL & $r / \wedge \Delta$. & r/arr & T/TE. \\
\hline & RMSE &.$/ 191$ & DTr/ & $\cdot / r \cdot r$ & $\cdot / r .$. & . MAr & TET &.$/ \ldots$ &.$/ 194$ & . & 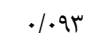 \\
\hline & RPD & q/9. & N/r山. & $q / V / f$ & 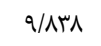 & $11 / .91$ & $|r / q| q$ & 19/9r & 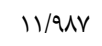 & ه/৭१९ & TM/AY. \\
\hline & AIC & $1 \pi r / D \cdot 9$ & $\mid K T / \cdot T F$ & שצא/rאו & $\mid K T / F V$ & IrE/V9D & ITH/AFD & G & ITK/KGV & $119 / \wedge \vee \Delta$ & $|r \Delta / q| \Lambda$ \\
\hline & BIC & VTr/או & $|r| / \lambda F \mid$ & KMT/TAK & $\mid r r / r q 4$ & זוצ/צr| & rوואואו & $\mid r \Delta / \Delta \Delta F$ & F & $119 / 994$ & צr/W/I \\
\hline \multirow[t]{7}{*}{$\Delta$} & R2 &.$/ 94$. & $\cdot / 9 \Lambda$ &.$/ 91$. &.$/ 989$ & $\cdot / 911$ & $\cdot / 918$ &.$/ 991$ &.$/ 9 \vee \wedge$ &.$/ 994$ &.$/ 994$ \\
\hline & VAF & १Q/TGY & ११/rद५ & १Q/T\&Y & $q \wedge / Q \subset \bar{T}$ & १Q/DHF & q৭/ᄉ. ए & १৭/৭९८ & 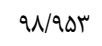 & $Q N / F+1$ & १৭/৭८ \\
\hline & MAPE & $\Gamma / F \mid \Lambda$ & r/r & r/r & T/KrA & $r / \&+q$ & $\Gamma / .9 \Delta$ & T/VAS & $r / r \cdot r$ & $\Gamma / \mp \wedge \Delta$ & T/QFV \\
\hline & RMSE & $\cdot / T \Delta S$ & $\cdot / T \Delta \Delta$ & $\cdot / T \Delta \Delta$ &.$/ 491$ & $. / Y \mid S$ & . /FT & .1 .91 &.$/ 499$ & $\cdot /$ TAV & . $/ \Delta T$ \\
\hline & RPD & $V / r \cdot G$ & V/rTr & V/rTV & s/TaD & Q/TEV & $|r / 4+|$ & TN/TNG & $s / \backslash \Delta \Lambda$ & r/^9т & एष/৭91 \\
\hline & AIC & $11 \mathrm{w} / \mathrm{v} \cdot 1$ & $11 \% / a \Lambda$. & $11 \% / a \Lambda$. & $11 \% / r / 9$ & $|r \Delta / \Delta T|$ & $\| V / r \& \Delta$ & $11 N / 948$ & 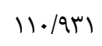 & $\| \digamma / \Delta \Delta$. & $\mid r \cdot / \| \Delta D$ \\
\hline & BIC & $1 \pi / \Delta 19$ & Irr/vqV & Irr/VqV & שאו/זו & גسץ/هזו & $I T V / M A T$ & WM/VG & $I T \cdot / V F \Lambda$ & N & $1 \mathrm{rq} / \mathrm{qVT}$ \\
\hline
\end{tabular}


ادامه جدول ب- شاخصهاى عملكرد (RPD, AIC, BIC, RMSE, VAF, MAPE and R2) مربوط به مدل در برداشت اول Continue of Table 3. Performance indices (RPD, AIC, BIC, RMSE, VAF, MAPE and R2) for models in first harvest

\begin{tabular}{|c|c|c|c|c|c|c|c|c|c|c|c|}
\hline \multirow[t]{7}{*}{7.5} & $\mathrm{R} 2$ &.$/ 9 \mathrm{VV}$ &.$/ 9 \mathrm{VV}$ &.$/ 9 \vee \Delta$ &.$/ 9 \mathrm{VV}$ &.$/ 99 \mathrm{~V}$ &.$/ 99$. &.$/ 990$ &.$/ 994$ &.$/ 991$ &.$/ 998$ \\
\hline & VAF & १९/०११ & ११/०११ & १९/ఎ४। & $99 / 099$ & QD/VTF & १৭/१५ळ & ११/१९. & १९/१人६ & $q N / \cdot r F$ & १৭/१९८ \\
\hline & MAPE & r/qr & r/qrT & $\Delta / \cdot r I$ & r/qr & $\Delta / I T$. & 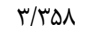 & $r / V I I$ & $r / \varphi \Delta$. & ఎ/ఎ१ఎ & T/DT \\
\hline & RMSE & $\cdot|| r \mid$ &.$/ 1 \mathrm{VI}$ & $\cdot|| 1 \mid$ &.$/|n|$ & $\cdot|\Delta|$. & $\cdot / \cdot v \cdot$ & . & عس.|. & ./MGV &.$/ \cdot \mathrm{TF}^{2}$ \\
\hline & RPD & $q / r \cdot V$ & $q / r \cdot V$ & N/vqu & $q / r \cdot v$ & $r / \Delta \cdot 1$ & משזא/M & $\Delta F / V I V$ & | & $p / q .$. & $9 N / \cdots q$ \\
\hline & AIC & N & $1 \& / K \varepsilon \mid$ & $\Lambda ৯ / V \cdot V$ & N & ع & $9 T / V \cdot 1$ & $94 / M M$ & 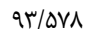 & $q F / V T F$ & QY/AKr \\
\hline & BIC & $q q / \cdot v q$ & $q \& / \cdot v q$ & QD/DTF & $q \& / \cdot v q$ & $\| F / \Delta \Delta F$ & $1 \cdot r / \Delta 1 \Lambda$ & $1+r / \cdots \Delta$ & 1. & $1 \cdot F / \Delta F \mid$ & $1+K / F D 1$ \\
\hline
\end{tabular}

جدول ع- شاخص هاى عملكرد (RPD, AIC, BIC, RMSE, VAF, MAPE and R2) مر بوط به مدل در برداشت دوم Table 4. Performance indices (RPD, AIC, BIC, RMSE, VAF, MAPE and R2) for models in second harvest

\begin{tabular}{|c|c|c|c|c|c|c|c|c|c|c|c|}
\hline 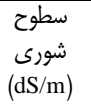 & شاخصهاى & PCR & PLS & OLS & LR & $\log R$ & NL R & NP R & MLP & $\mathrm{RBF}$ & ANFIS \\
\hline \multirow[t]{7}{*}{0} & $\mathrm{R} 2$ &.$/ 918$ &.$/ 918$ &.$/ 918$ &.$/ 918$ &.$/ 9 \vee \Delta$ &.$/ 911$ &.$/ 919$ & سM/9. &.$/ 990$ &.$/ 99$. \\
\hline & VAF & $99 / \Delta 11$ & qq/रq & $99 / \Delta 11$ & $99 / 0.9$ & $98 / 199$ & ११/४४ & ૧৭/А।V & $\Lambda \varepsilon / \mu \cdot \varphi$ & $q \wedge / \cdots q$ & 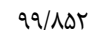 \\
\hline & MAPE & $m /|9|$ & $r / 19 q$ & $r /|q|$ & r/Mr & r/q६q & سM/qT & $r / 998$ & $p / q v^{c}$ & f/gVG & $\mathrm{T} / \mathrm{QVV}$ \\
\hline & RMSE & سצץ/. & $\cdot / \mathrm{rV}$. & سצץ/. & . /MGT & - /ATV &.$|49|$ & $\cdot|M F|$ & I/VAr & $\cdot / V 91$ & . \\
\hline & RPD & N/DQI & $N / F \mid \Delta$ & $N / 091$ & $\Lambda / \Delta \Delta \Lambda$ & ए/А११ & $11 / 998$ & gr/W/ & $1 / \Delta \cdot V$ & $r / q \uparrow q$ & IF/KT. \\
\hline & AIC & $r \cdot r / r / g$ & $r \cdot r / 19 q$ & $r \cdot r / r \mid g$ & rسז/r r & t. V/trt & $r \cdot r / \Delta V I$ & $r \cdot r / M I f r$ & $|V| / ৭ \Delta S$ & $r \cdots / 910$ & $r \cdot r / v q \Lambda$ \\
\hline & BIC & rr/r/r & $r|r / \cdot| r$ & سז./TIT & $r \mid r / \cdot D I$ & $r \mid V / \cdot r \cdot$ & rIr/rM & rIr/qrr & $1 \wedge 1 / V V^{m}$ & 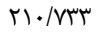 & rIF/gID \\
\hline \multirow[t]{7}{*}{$T / \Delta$} & $\mathrm{R} 2$ &.$/ 914$ &.$/ 91 r$ & ./9Ar &.$/ 915$ &.$/ 91$. &.$/ 91 \mathrm{f}$ &.$/ 911$ &.$/ 9 \wedge \mathrm{V}$ &.$/ 990$ &.$/ 919$ \\
\hline & VAF & 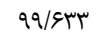 & ११/द्र & १९/द्राद & פ१/צח & ११/V।द & १९/А५ & 99/9५। & $99 / 9.9$ & $q \wedge / 9 \vee q$ & ११/१५ठ \\
\hline & MAPE & $r / v \cdots$ & TET & r/Vur & $r / v \cdots$ & r/rvq & $r / V V r$ & T/ETI & r/gr. & ए/А११ & T/TFE \\
\hline & RMSE & $\cdot / r \cdot r$ & $\cdot / T \cdot \Delta$ & $\cdot / r \cdot r$ & $\cdot / t \cdot r$ &.$/ 190$ &.$/ 1 F q$ & $\cdot / 1 \cdot 1$ &.$/ 111$ & $\cdot / R M$ &.$/ 1 \cdot r$ \\
\hline & RPD & q/9人र & q/Ais & $q / 9 \vee r$ & $9 / 9 \wedge \mathrm{F}$ & $1 \cdot / \Delta F$. & و & |N/qT| & $I V / T \cdot V$ & D/TH. & $r+/ 1 \ldots$ \\
\hline & AIC & ITE/ADT & Irg/qAV & $\mid r g / q \cdot V$ & ITE/ADT & r & IrV/arv & $|r|+|+|$ & $|r q / r|$. & Irg/AVT & $179 / 719$ \\
\hline & BIC & 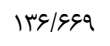 & ه. ه/ & IF/VTF & هوع/צr| & $\mid r \cdot / r \omega$. & IrV/VFe & $|r q / \wedge| \wedge$ & $\mid r q / \cdot r v$ & & צשמ/פשו \\
\hline \multirow[t]{7}{*}{$\Delta$} & $\mathrm{R} 2$ &.$/ 91$. &.$/ 91$. &.$/ 91$. &.$/ 91$. &.$/ 911$ &.$/ 918$ &.$/ 991$ &.$/ 910$ &.$/ 94 q$ & ./994 \\
\hline & VAF & १৭/rVน & ११/rV & ११/४९८ & १৭/rVน & १Q/DrF & $99 / 1.1$ & $99 / 9$ बद & १९/VT⿱ & ૧V/A. & १९/१८। \\
\hline & MAPE & r/Ta. & r/rq. & וIו & $r / T \Delta$. & T/DFT & $r / \cdot \Delta \Lambda$ & $r / V \Delta I$ & T/M & $\Delta / \backslash \notin \Lambda$ & $r / \Delta \Delta \omega$ \\
\hline & RMSE &.$|r q|$ & . & سצא/. & . & שTK/. &.$/ 1 F^{2}$ & $\cdot 1 \cdot v^{c}$ &.$/ 199$ & $\cdot / F \wedge r^{c}$ & $\cdot 1 \cdot \Delta V$ \\
\hline & RPD & V/rve & V/red & V/rII & V/rve & q/1V. & ه ब & 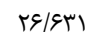 & W/זAた & r/^৭१ & $r F / v q$. \\
\hline & AIC & IN/NGY & $119 / \ldots$ & אשr/N/ & IN/NET & Irq/var & $|r| / Q \Delta V$ & $\mid r r / F V$. & $|r| / \Delta Q V$ & IIT/ATV & זسא/Fו \\
\hline & BIC & ITN/FVG & ITN/AIV & ITN/FAT & ITN/GVQ & $1 \mathrm{rq} / \Delta \mathrm{V}$. & IrI/var & IrT/TM & $1 r \cdot / 9 v a$ & ITr/gFt & ITY/IAT \\
\hline \multirow[t]{7}{*}{$V / \Delta$} & $\mathrm{R} 2$ &.$/ 9 \vee \wedge$ &.$/ 9 \mathrm{VV}$ &.$/ 9 v^{f}$ &.$/ 9 \mathrm{VV}$ &.$/ 981$ &.$/ 991$ &.$/ 990$ & ./994 & .1999 &.$/ 998$ \\
\hline & VAF & ११/ร५. & $99 / 8) 1$ & $99 / r \cdot r$ & १९/द५ & $৭ ৯ / \wedge \bowtie \wedge$ & ११/१४人 & ११/१८१ & $99 / 911$ & qא/rrq & $99 / 994$ \\
\hline & MAPE & $r / V \cdot q$ & r/VRr & $r / v \Lambda$. & r/Arی & $0 / \cdot 1 T$ & T/TAV & $r / q 91$ & $r / \cdot m r$ & $f /|f|$ & $r / c q q$ \\
\hline & RMSE &.$/ \mathrm{Vr}$ &.$/ 1 V^{e}$ & ה ג &.$/ 1 V^{e}$ & $\cdot / 011$ & $\cdot 1 \cdot V r$ & . & עc. & وسس/. & ב \\
\hline & RPD & Q/DTV & q/fer & $g|\Lambda \Lambda|$ & Q/FAV & $r / \Delta F \mid$ & rM/gVT & $0 \cdot / 9 \wedge \varepsilon$ & rq/eVr & t/vqp & $q \varepsilon / r \cdot r$ \\
\hline & AIC & $9 r / 11 V$ & q)/AVte & $q \cdot / V \& \wedge$ & חוגות & $1.9 / \Delta V$ & $Q \Lambda / r \Delta \Lambda$ & १९/VTr & ११/•५। & $M /{ }^{e} V^{e}$ & QN/AVT \\
\hline & BIC & 1. 1/quם & $1.1 / 991$ & $1 \cdots / \Delta \wedge \Delta$ & $1+1 / 8 \Delta 1$ & II9/rAV & $1 \cdot N / V q$ & $1+9 / \Delta \Delta$ & $1 \cdot$ N/NFq & qN/rqr & $1.1 / 9 q$. \\
\hline
\end{tabular}




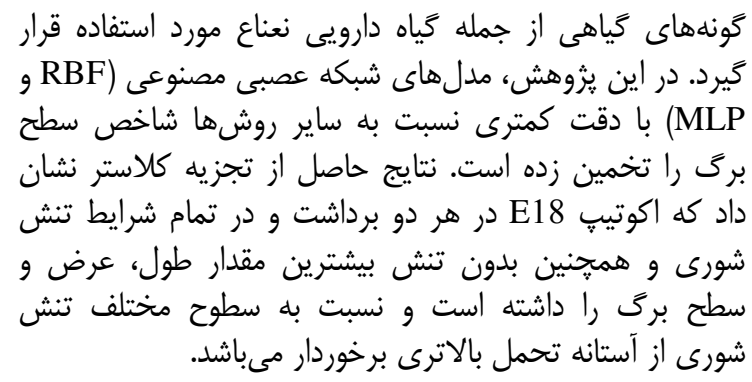

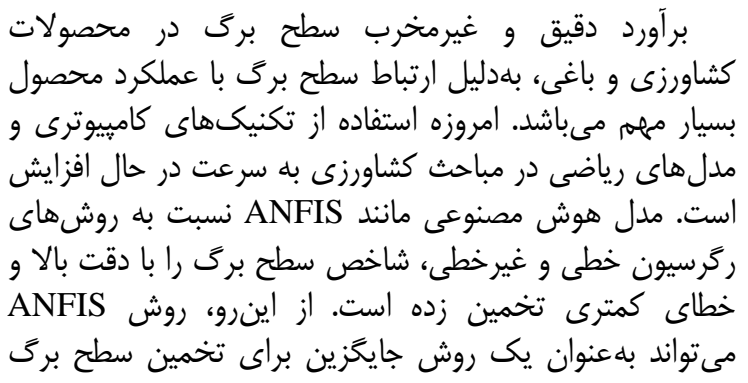

1. Aboukarim AM منابع prediction of the cotton crop leaf area. International Journal of Plant and Soil Science, 8(4): 1-13.

2. Ahmadian-Moghadam, H. 2012. Prediction of pepper (Capsicum annuum L.) leaf area using group method of data handling-type neural networks. International Journal of Agriculture Science, 2(11): 993999.

3. Amiri, M.J. and A. Shabani. 2017. Application of an Adaptive Neural-Based Fuzzy Inference System Model for Predicting Leaf Area. Communications in Soil Science and Plant Analysis, 48(14): 1669-1683.

4. Amiri, M.J., J. Abedi-Koupai, S.S. Eslamian, S.F. Mousavi and H. Hasheminejad. 2013. Modeling Pb (II) adsorption from aqueous solution by ostrich bone ash using adaptive neural-based fuzzy inference system. Journal of Environmental Science and Health, Part A 48: 543-558.

5. Aschonitis, V.G., D.M. Papamichail, A. Lithourgidis and E.A. Fano. 2014. Estimation of leaf area index and foliage area index of rice using an indirect gravimetric method. Communications in Soil Science and Plant Analysis, 45: 1726-1740.

6. Basbag, S., R. Ekinci and G. Oktay. 2008. Relationships between some physio-morphological traits and cotton (Gossypium hirsutum L.) yield. International Cotton Advisory Committee. Tenth Regional Meeting.

7. Blanco, F.F. and M.V. Folegatti. 2005. Estimation of leaf area for greenhouse cucumber by linear measurements under salinity and grafting. Scientia Agricola, 62(4): 305-309.

8. Caldas, L.S., C. Bravo, H. Piccolo and C.R. Faria. 1992. Measurement of leaf area with a hand-scanner linked to a microcomputer. Revista Brasileria de Fisiologia Vegetal, 4: 17-20.

9. Cargnelutti Filho, A., M. Toebe, B. Mendonça Alves, C. Burin and J.A. Kleinpaul. 2015. Estimation of leaf area of canola by leaf size. Bragantia, 74(2): 139-148.

10. Cargnelutti Filho, A., M. Toebe, C. Burin, A.L. Fick and G. Casarotto. 2012. Estimate of leaf area of forage turnip according to leaf dimensions. Bragantia, 71(1): 47-51.

11. Carvalho, J.O., M. Toebe, F.L. Tartaglia, C.T. Bandeira and A.L. Tambara. 2017. Leaf area estimation from linear measurements in different ages of Crotalaria juncea plants. Anais da Academia Brasileira de Ciências, 89(3): 1851-1868.

12. Cristofori, V., Y. Rouphael, E. Mendoza-de Gyves and C. Bignami. 2007. A simple model for estimating leaf area of hazelnut from linear measurements. Scientia Horticalture, 113(2): 221-225.

13. Daughtry, C.S.T. 1990. Direct measurements of canopy structure. International Journal of Remote Sensing Reviews, 5: 45-60.

14. De Aquino, L.A., V.C. dos Santos Júnior, J.V. Santos Guerra and M. Moreira Costa. 2011. Estimates of sunflower leaf area by a non-destructive method. Bragantia, 70(4): 832-836.

15. Ghanbari, S., A. Nooshkam, B.A. Fakheri and N. Mahdinezhad. 2019. Relationship between Yield and its Component in Soybean Genotypes (Glycine Max L.) using Multivariate Statistical Methods. Journal of Crop Breeding, 11(29): 85-92 (In Persian).

16. Ghorbani, H., H. Kashi, N. Hafezi Moghadas and S. Emamgholizadeh. 2015. Estimation of soil cation exchange capacity using multiple regression, artificial neural networks, and adaptive neuro-fuzzy inference system models in Golestan province, Iran. Communications in Soil Science and Plant Analysis, 46: 763-780.

17. Granier, C., C. Massonnet, O. Turc, B. Muller, K. Chenu and F. Tardieu. 2002. Individual leaf development in Arabidopsis thaliana: a stable thermal-time-based programme. Annals of Botany, 89(5): 595-604. 
18. Gupta, D.K., R. Prasad, P. Kumar, V. N. Mishra, P.K.S. Dikshit, S.B. Dwivedi and P.K. Srivastava. 2015. Crop variables estimation by adaptive neuro-fuzzy inference system using bistatic scatterometer data. In Microwave and Photonics (ICMAP), 2015 International Conference on 1-2 pp.

19. Hinnah, F., A. Heldwein, I. Maldaner, L. Loose, D.D. Lucas and M. Bortoluzzi. 2014. Estimation of eggplant leaf area from leaf dimensions. Bragantia, 73(3): 213-218.

20. Hosseini, M., S.A.M. Naeini, A.A. Dehghani and Y. Khaledian. 2016. Estimation of soil mechanical resistance parameter by using particle swarm optimization, genetic algorithm and multiple regression methods. Soil and Tillage Research, 157: 32-42.

21. Hosseini, M.S., D. Samsampour, M. Ebrahimi and M. Khanahmadi. 2019. Study of Physiological and Biochemical Changes of Iraninan Licorice (Glycyrrhiza Glabra) under Salinity Stress in Filed Condition. Journal of Crop Breeding, 11(29): 193-201 (In Persian).

22. Hosseini, M., S.R. Agereh, Y. Khaledian, H.J. Zoghalchali, E.C. Brevik and S.A. Naeini. 2017. Comparison of multiple statistical techniques to predict soil phosphorus. Applied Soil Ecology, 114: 123131.

23. Izadi, A., G. Ahmadvand, M. Asna Ashari and K. Piri. 2010. Effect of nitrogen and planting density on some growth characteristics, performance and essential oil in peppermint (Mentha piperita L.), Iranian Journal of Crop Research, 8(5): 824-836 (In Persian).

24. Kandiannan, K., U. Parthasarathy, K.S. Krishnamurthy, C.K. Thankamani and V. Srinivasan. 2009. Modeling individual leaf area of ginger (Zingiber officinale Roscoe) using leaf length and width. Scientia Horticulturae, 120(4): 532-537.

25. Kazem Alvandi R., A. Sharifan, M. Aghazadeh Meshghi. 2010. Investigation of the chemical composition and antimicrobial effect of essential oil of peppermint (Mentha piperita L.). Scientific Journal of Pathobiology, 4: 355- 364.

26. Keramatlou, I., M. Sharifani, H. Sabouri, M. Alizadeh and B. Kamkar. 2015. A simple linear model for leaf area estimation in Persian walnut (Juglans regia L.). Scientia Horticulturae, 184: 36-39.

27. Khaledian, Y., E.C. Brevik, P. Pereira, A. Cerdà, M.A. Fattah and H. Tazikeh. 2017. Modeling soil cation exchange capacity in multiple countries. Catena, 158: 194-200.

28. Khaledian, Y., J.N. Quinton, E.C. Brevik, P. Pereira and M. Zeraatpisheh. 2018. Developing global pedotransfer functions to estimate available soil phosphorus. Science of the Total Environment, 644: $1110-1116$.

29. Khoshnevisan, B., S. Rafiee, M. Omid and H. Mousazadeh. 2014. Development of an intelligent system based on ANFIS for predicting wheat grain yield on the basis of energy inputs. Information processing in agriculture, 1(1): 14-22.

30. Kumar, K., S. Kumar, V. Sankar, T. Sakthivel, G. Karunakaran and P.C. Tripathi. 2017. Non-destructive estimation of leaf area of durian (Durio zibethinus)-An artificial neural network approach. Scientia horticulturae, 219: 319-325.

31. Leroy, C., L. Saint-Andre and D. Auclair. 2007. Practical methods for non-destructive measurement of tree leaf area. Agroforestry systems, 71(2): 99-108.

32. Laio, F., G. Di Baldassarre and A. Montanari. 2009. Model selection techniques for the frequency analysis of hydrological extremes. Water Resources Research, 45(7): W07416.

33. Lizaso, J.I., W.D. Batchelor and M.E. Westgate. 2003. A leaf area model to simulate cultivar-specific expansion and senescence of maize leaves. Field Crops Research, 80: 1-17.

34. Mahmoudi, A. and M. Danesh. 2019. Assessment of Salinity Effects on Some Morphological and Physiological Traits and In Vitro Culture of Halophyte Plant (Salicornia Europaea). Journal of Crop Breeding, 11(29):161-168 (In Persian).

35. Marashi, M., A.M. Torkashvand, A. Ahmadi and M. Esfandyari. 2019. Adaptive neuro-fuzzy inference system: Estimation of soil aggregates stability. Acta Ecologica Sinica, 39(1): 95-101.

36. Mousavi, S.F. and M.J. Amiri. 2012. Modeling nitrate concentration of groundwater using adaptive neural-based fuzzy inference system. Soil Water Research, 7(2): 73-83.

37. Naderloo, L., R. Alimardani, M. Omid, F. Sarmadian, P. Javadikia, M.Y. Torabi and F. Alimardani. 2012. Application of ANFIS to predict crop yield based on different energy inputs. Measurement, 45(6): 14061413.

38. Nyakwende, E., C.J. Paull and J.G. Atherton. 1997. Non-destructive determination of leaf area in tomato plants using image processing. Journal of Horticulture Science, 72(2): 225-262.

39. Odabas, M.S., E. Ergun and F. Oner. 2013. Artificial neural network approach for the prediction of the corn (Zea mays L.) leaf area. Bulgarian Journal of Agricultural Science, 19(4): 766-769.

40. Olsoy, P.J., J.J. Mitchell, D.F. Levia, P.E. Clark and N.F. Glenn. 2016. Estimation of big sagebrush leaf area index with terrestrial laser scanning. Ecological indicators, 61: 815-821. 
41. Rodríguez Padrón, R.A., S.J. Lopes, A. Swarowsky, R.R. Cerquera, C.U. Nogueira and M. Maffei. 2016. Nondestructive models to estimate leaf area on bell pepper crop. Ciência Rural, 46(11): 1938-1944.

42. Ross, J., V. Ross and A. Koppel. 2000. Estimation of leaf area and its vertical distribution during growth period. Agricultural and Forest Meteorology, 101: 237-246.

43. Schwab, N.T., N.A. Streck, A. Rehbein, B.S. Ribeiro, L.O. Ulhmann, J.A. Langner and C.C. Becker. 2014. Linear dimensions of leaves and its use for estimating the vertical profile of leaf area in gladiolus. Bragantia, 73(2): 97-105.

44. Shabani, A., A.R. Sepaskhah and A.A. Kamgar-Haghighi. 2013. Growth and physiologic response of rapeseed (Brassica napus L.) to deficit irrigation, water salinity and planting method. International Journal of Plant Production, 7(3): 569-96.

45. Shabani, A., K.A. Ghaffary, A.R. Sepaskhah and A.A. Kamgar-Haghighi. 2017. Using the artificial neural network to estimate leaf area. Scientia Horticulturae, 216: 103-110.

46. Taherian, M., M.R. Bihamta, S.A. Peyghambari, H. Alizadeh and A. Rasoulnia. 2019. Stability Analysis and Selection of Salinity Tolerant barley Genotypes. Journal of Crop Breeding, 11(29): 93-103 (In Persian).

47. Toebe, M., A. Cargnelutti Filho, L.H. Loose, A.B. Heldwein and A.J. Zanon. 2012. Leaf area of snap bean (Phaseolus vulgaris L.) according to leaf dimensions. Semina: Ciências Agrárias, 33(1): 2491-2500.

48. Vazquez-Cruz, M.A., R. Luna-Rubio, L.M. Contreras-Medina, I. Torres-Pacheco and R.G. GuevaraGonzalez. 2012. Estimating the response of tomato (Solanum lycopersicum) leaf area to changes in climate and salicylic acid applications by means of artificial neural networks. Biosystems Engineering, 112(4): 319-327.

49. Yilmaz, I. and O. Kaynar. 2011. Multiple regression, ANN (RBF, MLP) and ANFIS models for prediction of swell potential of clayey soils. Expert Systems with Applications, 38: 5958-5966. 


\title{
Study of Diversity and Estimation of Leaf Area in Different Mint Ecotypes Using Artificial Intelligence and Regression Models under Salinity Stress Conditions
}

\author{
Seyed Jaber Hosseini ${ }^{1}$, Zeinolabedin Tahmasebi-Sarvestani ${ }^{2}$, Hematolahe Pirdashti ${ }^{3}$, \\ Seyed Ali Mohammad Modarres Sanavi ${ }^{4}$, Ali Mokhtassi-Bidgoli ${ }^{5}$ and Saeid Hazrati ${ }^{6}$ \\ 1- PhD. Student, Department of Agriculture, Tarbiat Modares University, Tehran, Iran \\ 2- Associate Professor, Department of Agriculture, Tarbiat Modares University, Tehran, Iran \\ (Corresponding author: tahmaseb@ modares.ac.ir) \\ 3- Professor, Sari Agricultural Sciences and Natural Resources University \\ 4 and 5- Professor and Assistant Professor, Department of Agriculture, Tarbiat Modarres University \\ 6- Assistant Professor, Department of Agronomy and Plant Breeding, Faculty of Agriculture, Azarbaijan Shahid Madani \\ University, Tabriz-Iran \\ Received: April 22, $2019 \quad$ Accepted: October 31, 2019
}

\begin{abstract}
Leaf area is a key indicator for the growth and production of plant products and also determines the efficiency of light consumption. Therefore, the study of diversity and also the estimation of leaf area in different mint ecotypes is particular importance. One of the common methods for estimating leaf area is regression analysis, the leaf area as independent variable, and leaf length and width as dependent variable. In this study, leaf area of 18 mint ecotypes with different models of ANFIS, artificial neural network (MLP and RBF), linear and nonlinear regressions using two inputs of leaf length and width in four levels of salinity stress (control, 2.5, 5 and $7.5 \mathrm{dS} / \mathrm{m}$ ) were estimated in two levels of harvesting. The results showed that there is a high correlation between length and width with leaf area, so that the width correlation with leaf area was greater than leaf length. Among the regression models in both harvest levels and at all levels of salinity stress, the NLR model is presented as the best and most accurate model. ANFIS model was harvested in both stages and at all levels of stress was more accurate than other models and less error rate was obtained. Also, the results of cluster analysis showed that there is a good variation between ecotypes. In addition, the mean comparison between different cluster analysis groups showed that the highest amount of length, width and leaf area was taken in both stages and at all levels of salinity stress was related to E18.
\end{abstract}

Keywords: Ecotypes, Leaf Area, Mint, Regression Models, Salinity Stress 